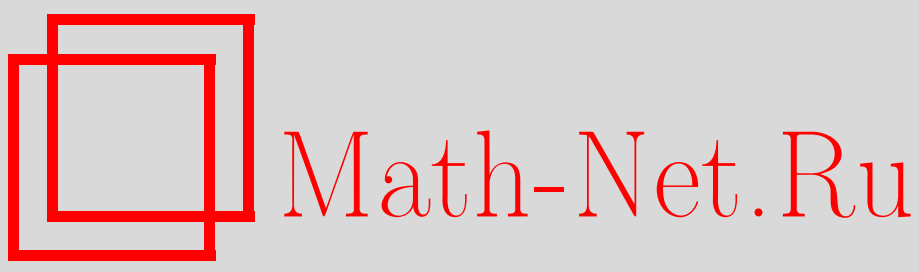

М. Ю. Планида, Асимптотики собственных элементов лапласиана с сингулярными возмущениями граничных условий на узких и тонких множествах, Матем. сб., 2005, том 196, номер 5, 83-120

DOI: https://doi.org/10.4213/sm1359

Использование Общероссийского математического портала Math-Net.Ru подразумевает, что вы прочитали и согласны с пользовательским соглашением

http://www.mathnet.ru/rus/agreement

Параметры загрузки:

IP : 54.174 .149 .18

26 апреля 2023 г., 15:02:02 
УДК 517.956

\author{
М. Ю. Планида
}

\title{
Асимптотики собственных элементов лапласиана с сингулярными возмущениями граничных условий на узких и тонких множествах
}

\begin{abstract}
Изучаются возмущения трехмерной задачи Дирихле в ограниченной области Первьй тип возмущений - смена типа граничного условия на узкой полоске, стягивающейся к замкнутой кривой на границе. Второй тип возмущений осуществляется вырезанием в области тонкого "тороидального" тела, стягивающегося также к замкнутой кривой (но уже лежащей внутри области), и заданием на границе этого тонкого тела граничного условия Неймана. Для этих задач методом согласования асимптотических разложений построены полные асимптотики по малому параметру собственных значений, сходящихся к простым собственным значениям невозмущенной задачи, и соответствующих собственньт функций. Малым параметром является соответственно ширина полоски и диаметр сечения тора.
\end{abstract}

Библиографияя: 45 названий.

\section{Введение}

Построению асимптотик решений эллиптических краевых задач в сингулярно возмушенных областях посвящено достаточно много работ (см., например, [1]-[15] и содержащийся в этих работах обзор литературы). Отдельньй интерес представляют асимптотики собственных значений таких краевых задач. Типичньми примерами, относящимися к этому классу, являются краевые задачи для оператора Лапласа в ограниченных областях с малыми отверстиями [16]-[20], с узкими щелями [21], с тонкими отростками и каналами связи [22]-[26], со сменой типа граничного условия на малой части границы, стягивающейся в точку [27]-[30], с часто меняющимся типом граничных условий [31]-[35].

В настояшей работе строятся полные асимптотики по малому параметру собственных значений и собственных функций оператора Лапласа для двух типов сингулярных возмущений граничных условий. А именно рассматриваются возмушения трехмерной задачи Дирихле, осушествляемые:

а) сменой типа граничного условия на узкой полоске, стягивающейся к замкнутой гладкой кривой на гранище (на границе узкой полоски задается условие Неймана);

Работа вьполнена при поддержке Российского фонда фундаментальных исследований (грант № 02-01-00693), Программы поддержки ведущих научных школ РФ (грант № НШ-1446.2003.1) и Министерства образования РФ (грант № А03-2.8-63). 
б) вырезанием в области тонкого “тороидального” тела, также стягивающегося к замкнутой кривой (но уже лежащей внутри области). На границе этого тонкого тела задается граничное условие Неймана.

Асимптотики строятся методом согласования асимптотических разложений [6]. Малым параметром является соответственно ширина полоски и диаметр сечения тора.

\section{§1. Формулировка основных результатов}

Всюду далее $x=\left(x_{1}, x_{2}, x_{3}\right), \Omega \subset \mathbb{R}^{3}$ - односвязная ограниченная область с гладкой границей $\Gamma, \gamma \in C^{\infty}$ - замкнутая кривая без самопересечений, лежащая либо на $\Gamma$, либо в $\Omega, s$ - натуральный параметр этой кривой, $0<\varepsilon \ll 1, \tau-$ вектор внутренней нормали, $(\cdot, \cdot)_{\Omega}$ - скалярное произведение в $L_{2}(\Omega),[\cdot, \cdot]-$ векторное произведение, $\lambda_{0}$ - простое собственное значение краевой задачи Дирихле

$$
-\Delta \psi_{0}=\lambda_{0} \psi_{0} \quad \text { в } \Omega, \quad \psi_{0}=0 \text { на } \Gamma,
$$

а $\psi_{0}$ - соответствуюшая нормированная в $L_{2}(\Omega)$ собственная функция.

В работе рассматриваются следующие задачи.

ЗАДАчА А1. Пусть $\gamma \subset \Gamma, \mathbf{R}(s, u)$ - параметрическое уравнение, $\mathbf{n}(s, u)-$ вектор внутренней нормали поверхности Г. Параметр и определим следующим образом. Через кажсдую точку кривой $\gamma$ перпендикулярно к ней проведем нормальную плоскость $\pi$. Обозначим $\widetilde{\gamma}=\Gamma \cap \pi, \quad u=y_{1}$ - натуральный параметр на $\widetilde{\gamma}$, причем $y_{1}=0$ соответствует точкам кривой $\gamma$. В окрестности $\gamma$ введем координаты $(y, s), y=\left(y_{1}, y_{2}\right)$, по правилу $x=\mathbf{R}\left(s, y_{1}\right)+y_{2} \mathbf{n}\left(s, y_{1}\right)$, әде $y_{2}$ - расстояние до $\gamma$ вдоль внутренней нормали. Положим $\gamma_{\varepsilon}=\left\{\left(y_{1}, 0, s\right): \varepsilon f_{1}(s)<y_{1}<\varepsilon f_{2}(s)\right\}, \quad \Gamma_{\varepsilon}=\Gamma \backslash \bar{\gamma}_{\varepsilon}$, әде $f_{1}(s), f_{2}(s) \in C^{\infty}(\gamma)$ (см. рис. 1). Будет исследована следующая краевая задача:

$$
-\Delta \psi_{\varepsilon}=\lambda_{\varepsilon} \psi_{\varepsilon} \quad \boldsymbol{в} \Omega, \quad \psi_{\varepsilon}=0 \quad \text { на } \Gamma_{\varepsilon}, \quad \frac{\partial \psi_{\varepsilon}}{\partial \tau}=0 \quad \text { на } \gamma_{\varepsilon}
$$

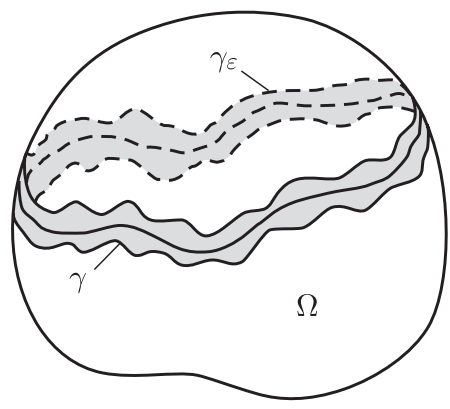

Рис. 1 
ЗАДАЧА А2. Пусть $\gamma \subset \Omega, \mathbf{r}=\mathbf{r}(s)$ - уравнение кривой, $\mathbf{t}(s)-$ единичный касательный вектор, $\mathbf{n}(s)$ - произвольное бесконечно дифференцируемое векторное поле единичных векторов нормали на кривой. На этой кривой зададим еще одно векторное поле $\mathbf{b}(s)$ по правилу $\mathbf{b}(s)=[\mathbf{t}(s), \mathbf{n}(s)]$. В каждой точке кривой будем рассматривать декартову систему координат, определяемую репером $(\mathbf{t}(s), \mathbf{n}(s), \mathbf{b}(s))$. Координать, связанные с векторами $\mathbf{n}(s)$ $u \mathbf{b}(s)$, обозначим через $y_{1}$ и $y_{2}$ соответственно. Тогда $x=\mathbf{r}(s)+y_{1} \mathbf{n}(s)+$ $y_{2} \mathbf{b}(s)$. Обозначим через $\omega$ односвязную ограниченную область в $\mathbb{R}^{2}$ с гладкой граничей, содержащую начало координат, $\omega_{\varepsilon}=\left\{y \in \mathbb{R}^{2}: \varepsilon^{-1} y \in \omega\right\}$, $\sigma_{\varepsilon}=\left\{x \in \mathbb{R}^{3}: s \in \gamma, y \in \omega_{\varepsilon}\right\}, \quad \Omega_{\varepsilon}=\Omega \backslash \bar{\sigma}_{\varepsilon}$ (см. рис. 2). Исследуется краевая задача вне тонкого тора:

$$
-\Delta \psi^{\varepsilon}=\lambda^{\varepsilon} \psi^{\varepsilon} \quad \text { в } \quad \Omega_{\varepsilon}, \quad \frac{\partial \psi^{\varepsilon}}{\partial \tau}=0 \quad \text { на } \partial \sigma_{\varepsilon}, \quad \psi^{\varepsilon}=0 \quad \text { на } \Gamma .
$$
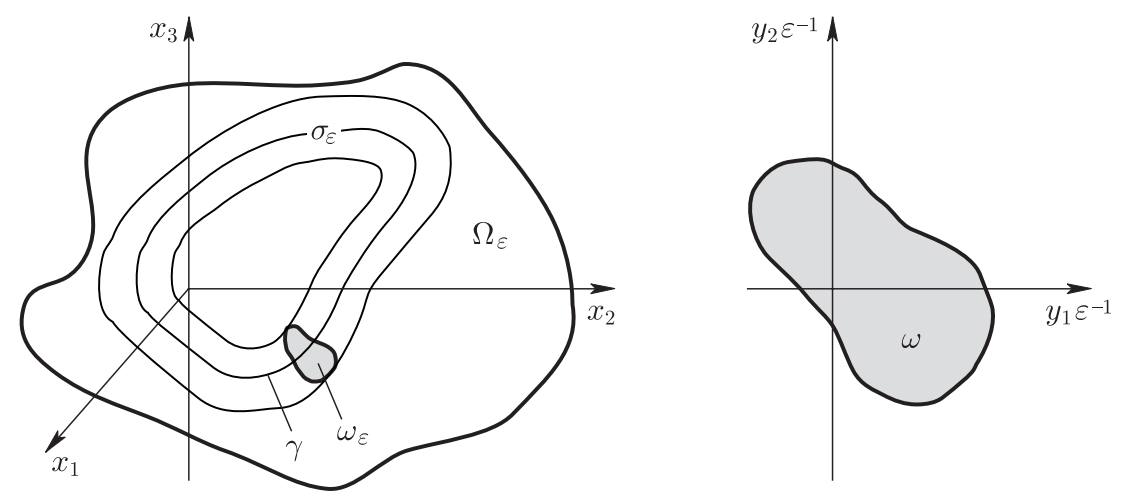

Рис. 2

Краевые задачи (1.2), (1.3) будем называть возмущенныции, а задачу Дирихле (1.1) - предельной.

В [36]-[38] были рассмотрены вопросы сходимости собственных значений и собственных функций возмущенных задач (1.2), (1.3). В частности, было доказано, что если $\lambda_{0}$ - простое собственное значение предельной задачи (1.1), то существует единственное и простое собственное значение возмушенной задачи, сходящееся при $\varepsilon \rightarrow 0$ к $\lambda_{0}$, а для нормированных в $L_{2}(\Omega)$ собственных функций имеет место сходимость в $L_{2}(\Omega)$ (собственная функция возмушенной задачи (1.3) считается продолженной нулем в $\Omega$ ).

Обозначим $S_{\mu}^{2}=\left\{x=\left(y_{1}, y_{2}, s\right): s \in \gamma,|y|<\mu\right\}$. Основным содержанием работы является доказательство следуюших утверждений.

Теорема 1.1. Пусть $\lambda_{0}$ - простое собственное значение предельной задачи (1.1), $\psi_{0}$ - соответствующая нормированная в $L_{2}(\Omega)$ собственная функчия. Тогда асимптотика собственного значения $\lambda_{\varepsilon}$ возмущенной задачи $(1.2)$, 
сходящегося $к \lambda_{0}$ при $\varepsilon \rightarrow 0$, имеет вид

$$
\begin{aligned}
\lambda_{\varepsilon} & =\lambda_{0}+\sum_{i=0}^{\infty} \sum_{j=0}^{[i / 2]} \varepsilon^{2+i} \ln ^{j} \varepsilon \lambda_{2+i, j}, \\
\lambda_{2,0} & =-\frac{\pi}{8} \int_{\gamma}\left(\frac{\partial \psi_{0}}{\partial \tau}\right)^{2}\left(f_{2}-f_{1}\right)^{2} d s, \\
\lambda_{2 t+2, t} & =-\frac{\pi}{8^{t+1}} \int_{\gamma} \frac{\partial \psi_{0}}{\partial \tau}\left(f_{2}-f_{1}\right)^{2} \mathscr{D}^{t} \frac{\partial \psi_{0}}{\partial \tau} d s,
\end{aligned}
$$

где D - линейный дифференциальный оператор второго порядка:

$$
\mathscr{D} u:=\left(\alpha(s) \frac{d^{2}}{d s^{2}}+\beta(s)\right)\left[\left(f_{2}-f_{1}\right)^{2} u\right]
$$

$\alpha(s), \beta(s) \in C^{\infty}(\gamma)$ зависят от геометрии $\gamma$ и $\Omega$. Асимптотики соответствующей собственной функиии $\psi_{\varepsilon}$ в норме $W_{2}^{1}$ имеют вид

$$
\begin{array}{ll}
\psi_{\varepsilon}(x)=\psi_{0}(x)+\sum_{i=0}^{\infty} \sum_{j=0}^{[i / 2]} \varepsilon^{2+i} \ln ^{j} \varepsilon \psi_{2+i, j}(x) & \text { в } \Omega \backslash S_{\varepsilon^{1 / 2}}^{2}, \\
\psi_{\varepsilon}(x)=\sum_{i=1}^{\infty} \sum_{j=0}^{[(i-1) / 2]} \varepsilon^{i} \ln ^{j} \varepsilon v_{i, j}\left(\frac{y}{\varepsilon} ; s\right) & \text { в } \Omega \cap S_{2 \varepsilon^{1 / 2}}^{2}
\end{array}
$$

где $v_{1,0}$ определяется в (2.7).

Заметим, что в случае, когда $\gamma$ - плоская кривая (например, $\gamma \subset \Omega \cap\left\{x_{3}=0\right\}$ ), имеем

$$
\alpha(s)=-\frac{1}{2}, \quad \beta(s)=-\frac{1}{2}\left(\frac{\kappa^{2}(s)}{4}+\lambda_{0}\right)
$$

где $|\kappa|-$ кривизна кривой $\gamma$. Для этого случая первый член асимптотики был построен в [39].

Теорема 1.2. Пусть $\lambda_{0}$ - простое собственное значение предельной задачи (1.1), $\psi_{0}$ - соответствующая нормированная в $L_{2}(\Omega)$ собственная функиия. Тогда асимптотика собственного значения $\lambda^{\varepsilon}$ возмущенной задачи (1.3), сходящегося $\kappa \lambda_{0}$ при $\varepsilon \rightarrow 0$, имеет вид (1.4), где

$$
\begin{aligned}
\lambda_{2,0}=|\omega| & \left.\int_{\gamma}\left(\lambda_{0} \psi_{0}^{2}-\left(\frac{\partial \psi_{0}}{\partial s}\right)^{2}\right)\right|_{y=0} d s \\
& -\left.\int_{\gamma} \nabla_{y} \psi_{0}\right|_{y=0} M \cdot\left(\left.\nabla_{y} \psi_{0}\right|_{y=0}+\left.\frac{1}{2} \mathbf{e}(s) \psi_{0}\right|_{y=0}\right) d s,
\end{aligned}
$$


$M$ - полохительно определенная симметричная $(2 \times 2)$-матрица из (3.18), зависящая от области $\omega$, вектор-функиия $\mathbf{e}(s) \in C^{\infty}(\gamma)$ зависит от геометрии кривой $\gamma$ и введенного на ней векторного поля $\mathbf{n}(s)$. Асимптотики соответствующей собственной функции $\psi^{\varepsilon}$ в норме $W_{2}^{1}$ имеют вид (1.8) ${ }_{\boldsymbol{B}} \Omega_{\varepsilon} \backslash S_{\varepsilon^{1 / 2}}^{2} u$

$$
\psi^{\varepsilon}(x)=\sum_{i=0}^{\infty} \sum_{j=0}^{[i / 2]} \varepsilon^{i} \ln ^{j} \varepsilon v_{i, j}\left(\frac{y}{\varepsilon} ; s\right) \quad \text { в } \Omega_{\varepsilon} \cap S_{2 \varepsilon^{1 / 2}}^{2},
$$

əде $v_{0,0}(y / \varepsilon ; s)=\left.\psi_{0}(x)\right|_{\gamma}$, а $v_{1,0}$ определяется в (3.19).

Заметим, что в случае, когда кривая $\gamma$ плоская, в формуле (1.10) вектор $\mathbf{e}(s)=$ $(0, \kappa(s)),|\kappa|-$ кривизна кривой $\gamma$. Для этого случая первый член асимптотики был построен в [38], [40].

Отметим, что в силу вариационных соображений в случае контактной задачи (1.2) имеет место неравенство $\lambda_{\varepsilon}-\lambda_{0}<0$, в то время как для задачи вне тонкой трубки (1.3) из (1.10) следует, что разность $\lambda_{\varepsilon}-\lambda_{0}$ не является знакоопределенной величиной. Подобное явление возникало в краевых задачах для оператора Лапласа в двумерных и трехмерных областях с вырезанным малым отверстием в случае, когда на границе отверстия задавалось условие Неймана, а отверстие стягивалось к точке [19], [20].

Структура работы вьглядит следующим образом. Во втором и третьем параграфах методом согласования асимптотических разложений строятся первые возмушенные члены асимптотики собственного значения возмушенных задач (1.2) и (1.3) соответственно. Коэффициенты рядов (1.8), (1.9), (1.11) являются сингулярными решениями некоторых модельных задач, не содержащих малый параметр $\varepsilon$. В $\S 4$ будут исследованы решения задачи Дирихле с заданными особенностями на кривой $\gamma$, необходимые при построении асимптотик собственных функций (1.8). В $\S 5$ будут найдены решения уравнения Пуассона в полуплоскости и в $\mathbb{R}^{2} \backslash \bar{\omega}$ с растушей правой частью, также необходимые при построении асимптотик собственных функций (1.9) и (1.11). В шестом параграфе доказывается теорема 1.1, а в седьмом - теорема 1.2 .

\section{§2. Построение первого члена асимптотики краевой задачи (1.2)}

В этом параграфе рассматривается задача А1. Методом согласования асимптотических разложений (на формальном уровне) строится первьй член теории возмушений собственного значения краевой задачи (1.2). В рассматриваемом случае гладкая кривая $\gamma$ лежит на границе Г области $\Omega,(y, s)$ - локальная система координат, заданная в окрестности кривой $\gamma$.

В силу граничного условия в (1.1) имеем

$$
\begin{aligned}
& \psi_{0}(x(y ; s))=\sum_{i=1}^{\infty} P_{i}(y ; s), \quad r=|y| \rightarrow 0 \\
& P_{1}(y ; s)=c_{01}(s) y_{2}, \quad c_{01}(s)=\left.\frac{\partial \psi_{0}}{\partial y_{2}}\right|_{y=0},
\end{aligned}
$$


где $P_{i}(y ; s)$ - однородные полиномы степени $i$ по переменной $y$ с коэффициентами из $C^{\infty}(\gamma)$

Так как, очевидно, $\psi_{0}$ не удовлетворяет граничному условию Неймана на $\gamma_{\varepsilon}$, то в окрестности $\gamma_{\varepsilon}$ введем внутреннее разложение, зависяшее от $\varepsilon$ и так называемых "внутренних" переменных $\xi=\left(\xi_{1}, \xi_{2}\right)$, где $\xi_{i}=y_{i} \varepsilon^{-1}$. Положим $\rho=|\xi|$.

Переписывая (2.1) в переменных $\xi$, получаем

$$
\psi_{0}(x)=\sum_{j=1}^{\infty} \varepsilon^{j} P_{j}(\xi ; s), \quad \varepsilon \rho \rightarrow 0
$$

Так как $\psi_{\varepsilon} \rightarrow \psi_{0}$ в $L_{2}(\Omega)$ при $\varepsilon \rightarrow 0$, то, следуя методу согласования асимптотических разложений [6] и учитывая (2.3), приближенное значение собственной функции $\psi_{\varepsilon}$ в малой окрестности кривой $\gamma$ будем искать в виде

$$
\begin{gathered}
\psi_{\varepsilon}(x)=\varepsilon v_{1}(\xi ; s)+\cdots, \\
v_{1}(\xi ; s)=P_{1}(\xi ; s)(1+o(1)), \quad \rho \rightarrow \infty .
\end{gathered}
$$

При малых $y$ оператор Лапласа в переменных $(y, s)$ имеет вид

$$
\Delta=\Delta_{y}+y_{2} L_{2}\left(y, s, \frac{\partial}{\partial y_{1}}, \frac{\partial}{\partial y_{2}}\right)+L_{1}\left(y, s, \frac{\partial}{\partial y_{1}}, \frac{\partial}{\partial y_{2}}\right) \frac{\partial}{\partial s}+L_{0}(y, s) \frac{\partial^{2}}{\partial s^{2}}
$$

где $L_{p}\left(y, s, \frac{\partial}{\partial y_{1}}, \frac{\partial}{\partial y_{2}}\right)$ - однородные относительно символов дифференцирования $\frac{\partial}{\partial y_{1}}, \frac{\partial}{\partial y_{2}}$ полиномы степени $p$, коэффициенты которых есть функции от $(y, s)$, бесконечно дифференцируемые в окрестности $\gamma$, а $L_{0}(y, s)$ - некоторая бесконечно дифференцируемая в окрестности $\gamma$ функция. Подставляя (2.5), (2.4) в (1.2), переходя в полученном равенстве к переменным $\xi$ и выписывая коэффициенты при одинаковых степенях $\varepsilon$, получаем следуюшую краевую задачу:

$$
\Delta_{\xi} v_{1}=0, \quad \xi_{2}>0, \quad v_{1}=0, \quad \xi \in \Gamma_{1}, \quad \frac{\partial v_{1}}{\partial \xi_{2}}=0, \quad \xi \in \gamma_{1}
$$

где $\gamma_{1}=\left\{\xi: f_{1}(s)<\xi_{1}<f_{2}(s), \xi_{2}=0\right\}, \Gamma_{1}=\left\{\xi: \xi_{2}=0\right\} \backslash \bar{\gamma}_{1}(\mathrm{~cm}$. рис. 3). Пусть $\zeta=\xi_{1}+\mathrm{i} \xi_{2}$, где i-мнимая единица, $(\rho, \varphi)$ - полярные координаты, соответствующие $\xi$. Легко видеть, что функция

$$
v_{1}(\xi ; s)=c_{01}(s) \operatorname{Im} \sqrt{\left(\zeta-f_{1}(s)\right)\left(\zeta-f_{2}(s)\right)}
$$

удовлетворяет (2.6) и имеет на бесконечности асимптотику

$$
v_{1}(\xi ; s)=P_{1}(\xi ; s)+\sum_{j=1}^{\infty} \beta_{j}(s) \rho^{-j} \sin (j \varphi), \quad \rho \rightarrow \infty
$$




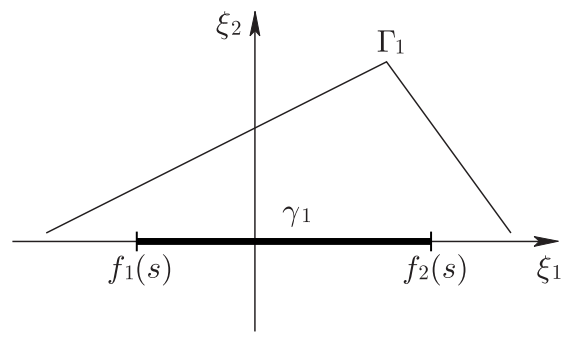

Рис. 3

где $\beta_{j}(s) \in C^{\infty}(\gamma)$ - некоторые явно вычисляемые функции и, в частности,

$$
\beta_{1}(s)=\frac{c_{01}(s)}{8}\left(f_{2}(s)-f_{1}(s)\right)^{2} .
$$

Переписьвая асимптотику $\varepsilon v_{1}$ при $\rho \rightarrow \infty$ в переменных $x$, получаем, что при $r \varepsilon^{-1} \rightarrow \infty$

$$
\psi_{\varepsilon}(x) \approx \varepsilon v_{1}(\xi ; s)=P_{1}(y ; s)+\sum_{j=1}^{\infty} \varepsilon^{j+1} \beta_{j}(s) r^{-j} \sin (j \varphi) .
$$

Отсюда в соответствии с методом согласования асимптотических разложений заключаем, что асимптотику собственной функции вне малой окрестности кривой $\gamma$ следует искать в виде

$$
\begin{aligned}
& \psi_{\varepsilon}(x)=\psi_{0}(x)+\varepsilon^{2} \psi_{2}(x)+\cdots, \\
& \psi_{2}(x)=\beta_{1}(s) r^{-1} \sin \varphi(1+o(1)), \quad r \rightarrow 0,
\end{aligned}
$$

а собственное значение $\lambda_{\varepsilon}$ в виде

$$
\lambda_{\varepsilon}=\lambda_{0}+\varepsilon^{2} \lambda_{2}+\cdots .
$$

Подставляя (2.11) и (2.9) в (1.2), приравнивая коэффициенты при степенях $\varepsilon^{m}$, $m \leqslant 2$, и переходя к пределу в описании $\Gamma_{\varepsilon}$, получаем краевую задачу (1.1) и краевую задачу для $\psi_{2}$ :

$$
-\Delta \psi_{2}=\lambda_{0} \psi_{2}+\lambda_{2} \psi_{0}, \quad x \in \Omega, \quad \psi_{2}=0, \quad x \in \Gamma \backslash \gamma
$$

В $\S 4$ будет доказано следующее утверждение.

Лемма 2.1. Пусть $a(s) \in C^{\infty}(\gamma)$. Тогда существует функиия $U^{(2)}(x ; a) \in$ $C^{\infty}(\bar{\Omega} \backslash \gamma)$, имеющая при $r \rightarrow 0$ дифференцируемую асимптотику

$$
U^{(2)}(x ; a(s))=r^{-1} a(s) \sin \varphi+O(1)
$$

и являющаяся решением краевой задачи (2.12) при $\lambda_{2}=\Lambda^{(2)}$, әде

$$
\Lambda^{(2)}=-\pi \int_{\gamma} c_{01}(s) a(s) d s
$$

Положим $\psi_{2}=U^{(2)}\left(x ; \beta_{1}(s)\right), \lambda_{2}=\Lambda^{(2)}$, где $\beta_{1}(s)$ определяется в (2.8). Тогда из леммы 2.1 следует, что функция $\psi_{2}$ является решением краевой задачи $(2.12)$ и имеет асимптотику (2.10). Из (2.13), (2.8) и (2.2) следует (1.5). 


\section{§3. Построение первого члена асимптотики краевой задачи (1.3)}

В этом параграфе рассматривается задача А2. Методом согласования асимптотических разложений (на формальном уровне) строится первьй член теории возмушений собственного значения краевой задачи (1.3). В рассматриваемом случае гладкая кривая $\gamma$ лежит в области $\Omega,(y, s)$ - локальная система координат, заданная в окрестности кривой $\gamma$. Обозначим

$$
c_{i j}(s)=\left.\frac{\partial^{i+j} \psi_{0}}{\partial y_{1}^{i} \partial y_{2}^{j}}\right|_{y=0} .
$$

Ясно, что при $r=|y| \rightarrow 0$

$$
\begin{gathered}
\psi_{0}(x(y ; s))=\sum_{i=0}^{\infty} P_{i}(y ; s), \\
P_{0}=c_{00}, \quad P_{1}=c_{10} y_{1}+c_{01} y_{2}, \quad P_{2}=\frac{c_{20} y_{1}^{2}+2 c_{11} y_{1} y_{2}+c_{02} y_{2}^{2}}{2},
\end{gathered}
$$

где, как и в предыдушем параграфе, $P_{i}(y ; s)$ - однородные полиномы степени $i$ по переменной $y$ с коэффициентами из $C^{\infty}(\gamma)$.

При малых $y$ оператор Лапласа в переменных $(y, s)$ можно записать в виде

$$
\begin{aligned}
\Delta= & \Delta_{y}-k_{1}(s) \frac{\partial}{\partial y_{1}}-k_{2}(s) \frac{\partial}{\partial y_{2}}+\frac{\partial^{2}}{\partial s^{2}}+Q_{2}(y ; s) L_{2}\left(y, s, \frac{\partial}{\partial y_{1}}, \frac{\partial}{\partial y_{2}}\right) \\
& +Q_{1}(y ; s) L_{1}\left(y, s, \frac{\partial}{\partial y_{1}}, \frac{\partial}{\partial y_{2}}\right) \frac{\partial}{\partial s}+y_{1} L_{0}(y, s) \frac{\partial^{2}}{\partial s^{2}},
\end{aligned}
$$

где $Q_{i}(y ; s)$ - однородные полиномы степени $i$ по переменной $y$ с коэффициентами из $C^{\infty}(\gamma), L_{p}\left(y, s, \frac{\partial}{\partial y_{1}}, \frac{\partial}{\partial y_{2}}\right)$ - однородные относительно символов дифференцирования $\frac{\partial}{\partial y_{1}}, \frac{\partial}{\partial y_{2}}$ полиномы степени $p$, коэффициенты которых есть функции от $(y, s)$, бесконечно дифференцируемые в окрестности $\gamma$, а $L_{0}(y, s)$ - некоторая бесконечно дифференцируемая в окрестности $\gamma$ функция, $k_{1}(s)=n(s) r^{\prime \prime}(s), k_{2}(s)=b(s) r^{\prime \prime}(s)$. Тогда в силу (1.1), (3.1), (3.4)

$$
c_{20}+c_{02}=k_{1} c_{10}+k_{2} c_{01}-c_{00}^{\prime \prime}-\lambda_{0} c_{00} .
$$

Как и в предыдушем параграфе, функция $\psi_{0}$ не удовлетворяет требуемым граничньм условиям на $\partial \sigma_{\varepsilon}$, поэтому асимптотику $\psi^{\varepsilon}$ в окрестности $\sigma_{\varepsilon}$ будем строить методом согласования асимптотических разложений в переменных $\xi$, где $\xi=\left(\xi_{1}, \xi_{2}\right), \xi_{i}=y_{i} \varepsilon^{-1}$. Переписывая $(3.2)$ в переменных $\xi$, получаем

$$
\psi_{0}(x)=\sum_{j=0}^{\infty} \varepsilon^{j} P_{j}(\xi ; s), \quad \varepsilon \rho \rightarrow 0 .
$$


Согласно методу согласования асимптотических разложений [6] и учитывая (3.6), приближенное значение собственной функции $\psi^{\varepsilon}$ в окрестности $\sigma_{\varepsilon}$ следует искать в виде

$$
\begin{gathered}
\psi^{\varepsilon}(x)=v_{0}(\xi ; s)+\varepsilon v_{1}(\xi ; s)+\varepsilon^{2} v_{2}(\xi ; s)+\cdots, \\
v_{i}(\xi ; s) \sim P_{i}(\xi ; s), \quad \rho \rightarrow \infty .
\end{gathered}
$$

Обозначим через $l_{\xi}$ натуральный параметр на $\partial \omega$, а через $\nu$ - внутреннюю нормаль к $\partial \omega$. Тогда

$$
\frac{\partial}{\partial \tau}=\varepsilon^{-1} \frac{\partial}{\partial \nu}-\sum_{i=1}^{\infty} \varepsilon^{i} p_{i}\left(l_{\xi}, s\right) \frac{\partial}{\partial s}
$$

где $p_{i}\left(l_{\xi}, s\right) \in C^{\infty}(\partial \omega \times \gamma)$.

Подставляя (3.4), (3.7), (3.9) в (1.3), переходя в полученном равенстве к переменным $\xi$ и выписывая коэффициенты при одинаковых степенях $\varepsilon$, получаем следуюшую краевую задачу:

$$
\Delta_{\xi} v_{0}=0, \quad \xi \in \mathbb{R}^{2} \backslash \bar{\omega}, \quad \frac{\partial v_{0}}{\partial \nu}=0, \quad \xi \in \partial \omega
$$

Очевидно, что функция

$$
v_{0}(\xi ; s) \equiv P_{0}(s)
$$

удовлетворяет (3.10) и (3.8). Подставляя далее (3.4), (3.7), (3.9) в (1.3), учитывая, что $v_{0}$ не зависит от $\xi$, получаем краевые задачи для $v_{1}$ и $v_{2}$ :

$$
\begin{gathered}
\Delta_{\xi} v_{1}=0, \quad \xi \in \mathbb{R}^{2} \backslash \bar{\omega}, \quad \frac{\partial v_{1}}{\partial \nu}=0, \quad \xi \in \partial \omega \\
\Delta_{\xi} v_{2}=k_{1} \frac{\partial v_{1}}{\partial \xi_{1}}+k_{2} \frac{\partial v_{1}}{\partial \xi_{2}}-\frac{\partial^{2} v_{0}}{\partial s^{2}}-\lambda_{0} v_{0}, \quad \xi \in \mathbb{R}^{2} \backslash \bar{\omega}, \quad \frac{\partial v_{2}}{\partial \nu}=0, \quad \xi \in \partial \omega .
\end{gathered}
$$

Исследуем краевые задачи (3.12), (3.13) на разрешимость и построим асимптотики $v_{1}, v_{2}$ при $\rho \rightarrow \infty$. Предварительно докажем следуюшее утверждение.

ЛЕмма 3.1. Если $Z_{n}(\xi ; s)$ - произвольный гармонический полином степени $n$ с коэффициентами из $C^{\infty}(\gamma)$, то существует гармоническая в $\mathbb{R}^{2} \backslash \bar{\omega}$ функция $V\left(\xi ; Z_{n}(\xi ; s)\right) \in C^{\infty}\left(\mathbb{R}^{2} \backslash \omega\right)$, удовлетворяющая однородным граничныцм условиям Неймана на $\partial \omega$, асимптотика которой при $\rho \rightarrow \infty$ имеет вид

$$
V\left(\xi ; Z_{n}(\xi ; s)\right)=Z_{n}(\xi ; s)+\sum_{j=1}^{\infty}\left(a_{j}(s) \sin (j \varphi)+b_{j}(s) \cos (j \varphi)\right) \rho^{-j}
$$

где $a_{j}, b_{j} \in C^{\infty}(\gamma)$. 
ДоказАТЕЛЬСТво. Функцию $V$ будем искать в виде $V\left(\xi ; Z_{n}(\xi ; s)\right)=Z_{n}(\xi ; s)+$ $\widetilde{v}(\xi ; s)$. Тогда для $\widetilde{v}$ получаем краевую задачу

$$
\Delta_{\xi} \widetilde{v}=0, \quad \xi \in \mathbb{R}^{2} \backslash \bar{\omega}, \quad \frac{\partial \widetilde{v}}{\partial \nu}=-\frac{\partial Z_{n}}{\partial \nu}, \quad \xi \in \partial \omega
$$

Из формулы Грина следует, что

$$
\int_{\partial \omega} \frac{\partial \widetilde{v}}{\partial \nu} d l_{\xi}=0
$$

Следовательно, существует решение краевой задачи (3.15), имеющее при $\rho \rightarrow \infty$ асимптотику

$$
\widetilde{v}(\xi ; s)=\sum_{j=1}^{\infty}\left(a_{j}(s) \sin (j \varphi)+b_{j}(s) \cos (j \varphi)\right) \rho^{-j},
$$

где $a_{j}, b_{j} \in C^{\infty}(\gamma)$. Таким образом, сушествует искомая функция $V$ с асимптотикой на бесконечности (3.14). Лемма доказана.

Непосредственно из данной леммы следует

СлЕДСТвИЕ 3.1. Существуют функции $V\left(\xi ; \xi_{1}\right), V\left(\xi ; \xi_{2}\right)$, гармонические в области $\mathbb{R}^{2} \backslash \bar{\omega}$ и удовлетворяющие однородныц граничным условиям Неймана на $\partial \omega$, асимптотика которых при $\rho \rightarrow \infty$ имеет вид

$$
V\left(\xi ; \xi_{i}\right)=\xi_{i}+\frac{1}{2 \pi} \sum_{j=1}^{2} m_{i j} \frac{\partial \ln \rho}{\partial \xi_{j}}+O\left(\rho^{-2}\right) .
$$

Покажем, что матрица $M=\left(m_{i j}\right)_{i, j=1,2}$ положительно определена и симметрична. Обозначим

$$
z_{i}=V\left(\xi ; \xi_{i}\right)-\xi_{i} .
$$

Из формулы Грина следует

$$
\begin{gathered}
\int_{\mathbb{R}^{2} \backslash \omega} \nabla z_{j} \cdot \nabla z_{p} d \xi=-\int_{\partial \omega} z_{j} \frac{\partial z_{p}}{\partial \nu} d l_{\xi}=-\int_{\partial \omega}\left(\xi_{j}+z_{j}\right) \frac{\partial z_{p}}{\partial \nu} d l_{\xi}-\int_{\partial \omega} \xi_{j} \frac{\partial \xi_{p}}{\partial \nu} d l_{\xi} \\
=-\lim _{R \rightarrow \infty} \int_{\partial S_{R}}\left\{\left(\xi_{j}+z_{j}\right) \frac{\partial z_{p}}{\partial \rho}-z_{p} \frac{\partial}{\partial \rho}\left(\xi_{j}+z_{j}\right)\right\} d l_{\xi}-\int_{\omega} \nabla \xi_{j} \cdot \nabla \xi_{p} d \xi .
\end{gathered}
$$

Отсюда в силу (3.16), (3.17) получаем

$$
\begin{aligned}
& \int_{\mathbb{R}^{2} \backslash \omega} \nabla z_{j} \cdot \nabla z_{p} d \xi=-\frac{1}{2 \pi} \lim _{R \rightarrow \infty} \int_{\partial S_{R}} \sum_{i=1}^{2} m_{p i}\left\{\xi_{j} \frac{\partial}{\partial \rho}\left(\frac{\partial \ln \rho}{\partial \xi_{i}}\right)-\frac{\partial \ln \rho}{\partial \xi_{i}} \frac{\partial \xi_{j}}{\partial \rho}\right\} d l_{\xi} \\
& -\delta_{p}^{j}|\omega|=m_{p j}-\delta_{p}^{j}|\omega|,
\end{aligned}
$$

где $\delta_{p}^{j}$ - символ Кронекера. Таким образом,

$$
M=E|\omega|+\left(\left(\nabla z_{j}, \nabla z_{p}\right)_{\mathbb{R}^{2} \backslash \omega}\right)_{j, p=1,2},
$$


где $E$ - единичная матрица, а матрица $M$ симметричная и зависит от области $\omega$. В силу критерия Сильвестра о положительной определенности квадратичных форм и неравенства Коши-Буняковского матрица $M$ положительно определенная.

В силу следствия 3.1 и (3.3) функция

$$
v_{1}(\xi ; s)=c_{10}(s) V\left(\xi ; \xi_{1}\right)+c_{01}(s) V\left(\xi ; \xi_{2}\right)
$$

является решением краевой задачи (3.12) и при $\rho \rightarrow \infty$ имеет асимптотику

$$
v_{1}(\xi ; s)=P_{1}^{(0)}(\xi ; s)+\sum_{j=1}^{\infty}\left(A_{j}(s) \sin j \varphi+B_{j}(s) \cos j \varphi\right) \rho^{-j},
$$

а следовательно, удовлетворяет (3.8), причем в силу (3.19), (3.16)

$$
A_{1}=\frac{1}{2 \pi}\left(c_{10} m_{12}+c_{01} m_{22}\right), \quad B_{1}=\frac{1}{2 \pi}\left(c_{10} m_{11}+c_{01} m_{21}\right) .
$$

Заметим, что из (3.17) и (3.19) следует

$$
v_{1}(\xi ; s)=P_{1}(\xi ; s)+c_{10} z_{1}+c_{01} z_{2} .
$$

Рассмотрим краевую задачу (3.13). Положим

$$
v_{2}=\frac{k_{1}}{2} \xi_{1} v_{1}+\frac{k_{2}}{2} \xi_{2} v_{1}-\frac{\rho^{2}}{4}\left(c_{00}^{\prime \prime}+\lambda_{0} c_{00}\right)+\widetilde{v}_{2} .
$$

Так как $\frac{\partial v_{1}}{\partial \nu}=0$ на $\partial \omega$, то в силу $(3.23),(3.13),(3.12),(3.11)$ краевая задача для $\widetilde{v}_{2}(\xi ; s)$ имеет вид

$$
\begin{gathered}
\Delta_{\xi} \widetilde{v}_{2}=0, \quad \xi \in \mathbb{R}^{2} \backslash \bar{\omega} \\
\frac{\partial \widetilde{v}_{2}}{\partial \nu}=-\frac{k_{1}}{2} v_{1} \frac{\partial \xi_{1}}{\partial \nu}-\frac{k_{2}}{2} v_{1} \frac{\partial \xi_{2}}{\partial \nu}+\frac{1}{4} \frac{\partial \rho^{2}}{\partial \nu}\left(c_{00}^{\prime \prime}+\lambda_{0} c_{00}\right), \quad \xi \in \partial \omega .
\end{gathered}
$$

Заметим, что из (3.3), (3.5), (3.8) и правой части (3.23) следует, что асимптотика $\widetilde{v}_{2}$ при $\rho \rightarrow \infty$ должна иметь вид $\widetilde{v}_{2}(\xi ; s)=Z_{2}(\xi ; s)+o\left(\rho^{2}\right)$. Будем искать $\widetilde{v}_{2}$ в виде

$$
\widetilde{v}_{2}(\xi ; s)=V\left(\xi ; Z_{2}(\xi ; s)\right)+\widehat{v}_{2}(\xi ; s), \quad \widehat{v}_{2}(\xi ; s)=o(\rho), \quad \rho \rightarrow \infty .
$$

Из (3.25) и (3.24) следует, что краевая задача для $\widehat{v}_{2}(\xi ; s)$ имеет вид

$$
\begin{gathered}
\Delta_{\xi} \widehat{v}_{2}=0, \quad \xi \in \mathbb{R}^{2} \backslash \bar{\omega}, \\
\frac{\partial \widehat{v}_{2}}{\partial \nu}=-\frac{k_{1}}{2} v_{1} \frac{\partial \xi_{1}}{\partial \nu}-\frac{k_{2}}{2} v_{1} \frac{\partial \xi_{2}}{\partial \nu}+\frac{1}{4} \frac{\partial \rho^{2}}{\partial \nu}\left(c_{00}^{\prime \prime}+\lambda_{0} c_{00}\right), \quad \xi \in \partial \omega .
\end{gathered}
$$

Известно (см., например, [41]), что существует решение $\widehat{v}_{2} \in C^{\infty}\left(\mathbb{R}^{2} \backslash \omega\right)$ краевой задачи (3.26) с асимптотикой при $\rho \rightarrow \infty$

$$
\widehat{v}_{2}(\xi ; s)=G(s) \ln \rho+\sum_{j=1}^{\infty} \rho^{-j}\left(\widetilde{a}_{j}(s) \sin (j \varphi)+\widetilde{b}_{j}(s) \cos (j \varphi)\right),
$$


где

$$
G(s)=\frac{1}{2 \pi} \int_{\partial \omega} \frac{\partial \widehat{v}_{2}}{\partial \nu} d l_{\xi}
$$

С другой стороны, в силу краевого условия в (3.26) имеем

$$
\int_{\partial \omega} \frac{\partial \widehat{v}_{2}}{\partial \nu} d l_{\xi}=-\frac{k_{1}}{2} \int_{\partial \omega} v_{1} \frac{\partial \xi_{1}}{\partial \nu} d l_{\xi}-\frac{k_{2}}{2} \int_{\partial \omega} v_{1} \frac{\partial \xi_{2}}{\partial \nu} d l_{\xi}+\frac{c_{00}^{\prime \prime}+\lambda_{0} c_{00}}{4} \int_{\partial \omega} \frac{\partial \rho^{2}}{\partial \nu} d l_{\xi} .
$$

Интегрируя по частям $\Delta_{\xi} \rho^{2}$ по области $\omega$, получаем

$$
\int_{\partial \omega} \frac{\partial \rho^{2}}{\partial \nu} d l_{\xi}=4|\omega|
$$

Из (3.22) следует, что

$$
\int_{\partial \omega} v_{1} \frac{\partial \xi_{1}}{\partial \nu} d l_{\xi}=\int_{\partial \omega} P_{1} \frac{\partial \xi_{1}}{\partial \nu} d l_{\xi}+\int_{\partial \omega}\left(c_{10} z_{1}+c_{01} z_{2}\right) \frac{\partial \xi_{1}}{\partial \nu} d l_{\xi}
$$

где функции $z_{i}$ гармонические в $\mathbb{R}^{2} \backslash \bar{\omega}$ и удовлетворяют на $\partial \omega$ краевому условию $\frac{\partial z_{i}}{\partial \nu}=-\frac{\partial \xi_{i}}{\partial \nu}$ в силу (3.17). Интегрируя по частям $\xi_{1} \Delta_{\xi} z_{i}$ по области $S_{R} \backslash \omega$ и учитывая асимптотики $z_{i}$ при $\rho \rightarrow \infty$ (см. (3.16), (3.17)), выводим

$$
\int_{\partial \omega} z_{1} \frac{\partial \xi_{1}}{\partial \nu} d l_{\xi}=m_{11}-|\omega|+O\left(R^{-1}\right), \quad \int_{\partial \omega} z_{2} \frac{\partial \xi_{1}}{\partial \nu} d l_{\xi}=m_{21}+O\left(R^{-1}\right) .
$$

Тогда

$$
\int_{\partial \omega}\left(c_{10} z_{1}+c_{01} z_{2}\right) \frac{\partial \xi_{1}}{\partial \nu} d l_{\xi}=c_{10} m_{12}+c_{01} m_{22}-|\omega| c_{10}+O\left(R^{-1}\right) .
$$

Из формулы Грина, примененной к области $\omega$, и (3.3) следует, что

$$
\int_{\partial \omega} P_{1} \frac{\partial \xi_{1}}{\partial \nu} d l_{\xi}=\int_{\partial \omega} \xi_{1}\left(c_{10} \frac{\partial \xi_{1}}{\partial \nu}+c_{01} \frac{\partial \xi_{2}}{\partial \nu}\right) d l_{\xi}=c_{10}|\omega| .
$$

Таким образом, в силу (3.31)-(3.33) имеем

$$
\int_{\partial \omega} v_{1} \frac{\partial \xi_{1}}{\partial \nu} d l_{\xi}=c_{10} m_{11}+c_{01} m_{21}+O\left(R^{-1}\right) .
$$

Аналогично можно показать, что

$$
\int_{\partial \omega} v_{1} \frac{\partial \xi_{2}}{\partial \tau} d l_{\xi}=c_{10} m_{21}+c_{01} m_{22}+O\left(R^{-1}\right) .
$$

Таким образом, из (3.29), (3.30), (3.34), (3.35) выводим

$$
\begin{aligned}
\int_{\partial \omega} \frac{\partial \widehat{v}_{2}}{\partial \nu} d l_{\xi}= & -\frac{k_{1}}{2}\left(c_{10} m_{11}+c_{01} m_{21}\right)-\frac{k_{2}}{2}\left(c_{10} m_{12}+c_{01} m_{22}\right) \\
& +|\omega|\left(c_{00}^{\prime \prime}+\lambda_{0} c_{00}\right)+O\left(R^{-1}\right) .
\end{aligned}
$$

Переходя к пределу при $R \rightarrow \infty$ в последнем равенстве, в силу (3.28) имеем

$$
G=-\frac{k_{1}}{4 \pi}\left(c_{10} m_{11}+c_{01} m_{21}\right)-\frac{k_{2}}{4 \pi}\left(c_{10} m_{12}+c_{01} m_{22}\right)+\frac{1}{2 \pi}|\omega|\left(c_{00}^{\prime \prime}+\lambda_{0} c_{00}\right) \text {. }
$$

Таким образом, из (3.23), (3.25), (3.27) следует, что краевая задача (3.13) имеет решение $v_{2}$ с асимптотикой

$$
v_{2}(\xi ; s)=P_{2}(\xi ; s)+G(s) \ln \rho+O(1), \quad \rho \rightarrow \infty,
$$

а следовательно, удовлетворяет (3.8). 
ЗАмЕчАниЕ 3.1. Отметим, что при переписывании асимптотики функции $\varepsilon^{2} v_{2}$ при $\rho \rightarrow \infty$ в переменных $x$ возникает слагаемое $\left(-\varepsilon^{2} \ln \varepsilon G(s)\right)$. Чтобы его уничтожить, введем во внутреннем разложении дополнительное слагаемое $\varepsilon^{2} \ln \varepsilon v_{2,1}$ с асимптотикой

$$
v_{2,1}(\xi ; s)=G(s)+o(1) .
$$

Следовательно, главные члены асимптотики $\psi^{\varepsilon}$ надо строить в виде

$$
\psi^{\varepsilon}(x)=v_{0}(\xi ; s)+\varepsilon v_{1}(\xi ; s)+\varepsilon^{2} \ln \varepsilon v_{2,1}(\xi ; s)+\varepsilon^{2} v_{2}(\xi ; s)+\cdots .
$$

Подставляя (3.39), (3.4), (3.9) в (1.3), переходя в полученном равенстве к переменньм $\xi$ и выписывая коэффициенты при $\varepsilon^{2} \ln \varepsilon$, получаем следуюшую краевую задачy:

$$
\Delta_{\xi} v_{2,1}=0, \quad \xi \in \mathbb{R}^{2} \backslash \bar{\omega}, \quad \frac{\partial v_{2,1}}{\partial \nu}=0, \quad \xi \in \partial \omega .
$$

Очевидно, что

$$
v_{2,1}(\xi ; s) \equiv G(s)
$$

является решением краевой задачи (3.40) и имеет асимптотику (3.38). Подставляя в (3.39) вместо $v_{i}$ их асимптотики на бесконечности $(3.11),(3.20),(3.37),(3.41)$ и переписывая последние в переменных $y$, получаем, что при $r \varepsilon^{-1} \rightarrow \infty$

$\psi^{\varepsilon}(\xi ; s)=\sum_{j=0}^{2} P_{j}(y ; s)+\varepsilon^{2}\left(r^{-1}\left(A_{1} \sin \varphi+B_{1} \cos \varphi\right)+G(s) \ln r\right)+O\left(\varepsilon^{2}+\varepsilon^{3} r^{-1}\right)$.

Следуя методу согласования асимптотических разложений, будем искать собственные элементы вне малой окрестности кривой $\gamma$ в виде $(2.9),(2.11)$, причем

$$
\psi_{2}(x)=r^{-1}\left(A_{1}(s) \sin \varphi+B_{1}(s) \cos \varphi\right)+G(s) \ln r+O(1), \quad r \rightarrow 0 .
$$

Подставляя (2.11) и (2.9) в (1.3), приравнивая коэффициенты при степенях $\varepsilon^{m}$, $m \leqslant 2$, и переходя к пределу в описании $\Omega_{\varepsilon}$, получаем краевую задачу (1.1) и краевую задачу для $\psi_{2}$ :

$$
-\Delta \psi_{2}=\lambda_{0} \psi_{2}+\lambda_{2} \psi_{0}, \quad x \in \Omega \backslash \gamma, \quad \psi_{2}=0, \quad x \in \Gamma .
$$

В $\S 4$ будет доказано следуюшее утверждение.

Лемма 3.2. Пусть $a(s), b(s), g(s) \in C^{\infty}(\gamma)$. Тогда существуют функции $U^{(0)}(x ; g), U^{(1)}(x ; a, b) \in C^{\infty}(\bar{\Omega} \backslash \gamma)$, имеющие при $r \rightarrow 0$ дифференцируемье асимптотики

$$
U^{(0)}(x ; g(s)) \sim g(s) \ln r, \quad U^{(1)}(x ; a(s), b(s)) \sim r^{-1}(a(s) \sin \varphi+b(s) \cos \varphi)
$$

и являющиеся решениями краевой задачи (3.43) при $\lambda_{2}=\Lambda^{(0)}$ и $\lambda_{2}=\Lambda^{(1)}$ соответственно, где

$$
\begin{aligned}
& \Lambda^{(0)}=2 \pi \int_{\gamma} c_{00} g d s, \\
& \Lambda^{(1)}=-2 \pi \int_{\gamma}\left(c_{10} b+c_{01} a\right) d s .
\end{aligned}
$$


Положим

$$
\psi_{2}(x)=U^{(0)}(x ; G(s))+U^{(1)}\left(x ; A_{1}(s), B_{1}(s)\right),
$$

где $A_{1}, B_{1}$ - коэффициенты из $(3.20)$, а $G$ - коэффициент из (3.36). Тогда функция $\psi_{2}$ является решением краевой задачи (3.43) при

$$
\lambda_{2}=\Lambda^{(1)}+\Lambda^{(0)}
$$

и при $r \rightarrow 0$ имеет асимптотику (3.42). Из (3.44)-(3.46), (3.36) и (3.21) следует (1.10).

\section{$\S 4$. Решения задачи Дирихле с особенностями на кривой $\gamma$}

Обозначим через $M_{p, q}(y, s, D), R_{p, q}(y, s, D)$ полиномы, однородные относительно $y$ и относительно символа дифференцирования $D$, с коэффициентами из $C^{\infty}(\gamma)$. Степень их однородности относительно $y$ равна $p$, а относительно $D$ равна $q$. Положим

$$
\begin{aligned}
& R_{i, 0}(y, s, D)=M_{i, 2}\left(y, s, D_{s}\right)+M_{i, 1}\left(y, s, D_{s}\right) \\
& R_{i, 1}(y, s, D)=M_{i, 1}\left(y, s, D_{s y}\right)+M_{i, 1}\left(y, s, D_{y}\right), \\
& R_{i, 2}(y, s, D)=M_{i, 2}\left(y, s, D_{y}\right)
\end{aligned}
$$

где $D_{y}=\left(\frac{\partial}{\partial y_{1}}, \frac{\partial}{\partial y_{2}}\right), D_{s}=\frac{\partial}{\partial s}, D_{s y}=\left(\frac{\partial^{2}}{\partial s \partial y_{1}}, \frac{\partial^{2}}{\partial s \partial y_{2}}\right)$. С учетом введенных обозначений оператор Лапласа в локальных переменных (в обеих задачах А1 и А2) можно записать в виде

$$
\Delta=\Delta_{y}-\sum_{i=1}^{\infty} R_{i, 2}(y, s, D)-\sum_{i=0}^{\infty} R_{i, 1}(y, s, D)-\sum_{i=0}^{\infty} R_{i, 0}(y, s, D) .
$$

В этом параграфе исследуются решения задачи Дирихле с заданными особенностями на кривой $\gamma$.

Вначале для случая, когда кривая $\gamma$ лежит на границе области $\Omega$ (т.е. для случая A1), рассмотрим следующую краевую задачу:

$$
-\left(\Delta+\lambda_{0}\right) U=F+\Lambda \psi_{0}, \quad x \in \Omega, \quad U=0, \quad x \in \Gamma \backslash \gamma,
$$

где $F$ - функция, имеющая особенность на $\gamma$. Будем исследовать краевую задачу (4.2), следуя [6].

Обозначим через $Z_{i}(y ; s)$ однородные гармонические полиномы степени $i$ по переменной $y$ с коэффициентами из $C^{\infty}(\gamma) ; P_{i}(y ; s), Q_{i}(y ; s)$ - однородные полиномы степени $i$ по переменной $y$ с коэффициентами из $C^{\infty}(\gamma) ; \Phi(i, j)-$ функции вида $\Phi(i, j)=r^{i} Z_{j}$

Доказательство следующих трех утверждений имеется в [6].

ЛЕмма 4.1. Для любого натурального $k$ и любого иелого $\alpha$ такого, что $(\alpha+2)(\alpha+2+2 k) \neq 0$, уравнение $\Delta_{y} u=\Phi(\alpha, k)$ имеет решение $u=\Phi(\alpha+2, k)$. Если жее $(\alpha+2)(\alpha+2+2 k)=0$, то это уравнение имеет решение $u=$ $\Phi(\alpha+2, k) \ln r$.

Для любого натурального $k$ и любого челого $\beta$ уравнение $\Delta_{y} u=\Phi(\beta, k) \ln r$ имеет решение $u=\Phi(\beta+2, k)(\ln r+a)$, где а - некоторая явно вычисляемая постоянная. 
Лемма 4.2. Для любого $Q_{k}(y ; s)$ справедливо единственное представление

$$
Q_{k}(y ; s)=\sum_{j=0}^{[k / 2]} \Phi(2 j, k-2 j) .
$$

Лемма 4.3. Для любых $Z_{k}(y ; s), Q_{j}(y ; s), k>j$, справедливо равенство

$$
Z_{k}(y ; s) Q_{j}(y ; s)=\sum_{l=0}^{j} \Phi(2 l, k+j-2 l) .
$$

С учетом лемм 4.2, 4.3 непосредственньми вычислениями легко проверить следуюшие равенства:

$$
\begin{aligned}
& R_{q, 2}(y, s, D) \Phi(i, j)=(i+2 j) \sum_{l=0}^{q} \Phi(2 l+i-2, q+j-2 l) \\
& \quad+\sum_{l=0}^{q} \Phi(2 l+i-4, q+j+2-2 l)+(i+2 j) \sum_{l=0}^{q} \Phi(2 l+i, q+j-2-2 l), \\
& R_{q, 1}(y, s, D) \Phi(i, j)=\sum_{l=0}^{q} \Phi(2 l+i-2, q+1+j-2 l) \\
& \quad+(i+2 j) \sum_{l=0}^{q} \Phi(2 l+i, q+j-2-2 l) \\
& \quad \\
& R_{q, 0}(y, s, D) \Phi(i, j)=\sum_{l=0}^{q} \Phi(2 l+i, q+j-2 l) \\
& R_{q, 2}(y, s, D)(\Phi(i, j) \ln r)=\ln r R_{q, 2}(y, s, D) \Phi(i, j)+R_{q, 2}(y, s, D) \Phi(i, j) \\
& R_{q, 1}(y, s, D)(\Phi(i, j) \ln r)=\ln r R_{q, 1}(y, s, D) \Phi(i, j)+R_{q, 1}(y, s, D) \Phi(i, j) \\
& R_{q, 0}(y, s, D)(\Phi(i, j) \ln r)=\ln r R_{q, 0}(y, s, D) \Phi(i, j) \\
& R_{q, 2}(y, s, D)(\Phi(i, j) \varphi)=\varphi R_{q, 2}(y, s, D) \Phi(i, j)+R_{q, 2}(y, s, D) \Phi(i, j) \\
& R_{q, 1}(y, s, D)(\Phi(i, j) \varphi)=\varphi R_{q, 1}(y, s, D) \Phi(i, j)+R_{q, 1}(y, s, D) \Phi(i, j) \\
& R_{q, 0}(y, s, D)(\Phi(i, j) \varphi)=\varphi R_{q, 0}(y, s, D) \Phi(i, j)
\end{aligned}
$$

С учетом (4.3) и леммы 4.3 непосредственными вычислениями легко доказать следуюшее утверждение.

Лемма 4.4. Для любого натурального $k$ и любого челого $\beta$ уравнение $\Delta_{y} u=$ $\Phi(\beta, k) \varphi$ имеет решение $u=\Phi(\beta+2, k)(\varphi+b)$, где $b$ - некоторая явно вычисляемая постоянная.

Пусть $\tilde{\mathscr{A}}_{k}$-множество рядов вида

$$
\widetilde{U}(y ; s)=\sum_{i=0}^{\infty} u_{i}(y ; s),
$$


где $u_{i}$ обрашаются в нуль при $y_{2}=0, y \neq 0$, и имеют вид

$$
\begin{aligned}
u_{0}(y ; s)= & r^{-2 k} Z_{k}(y ; s), \\
u_{i}(y ; s)= & \sum_{j=0}^{i} \Phi(-2 k+2 j-2, k+i-2 j+2), \quad 1 \leqslant i \leqslant k, \\
u_{k+i}(y ; s)= & \sum_{j=0}^{k} \Phi(-2 k+2 j-2,2 k+i-2 j+2)+\ln r \sum_{j=0}^{[i / 2]} \Phi(2 j, i-2 j) \\
& +\sum_{j=0}^{[i / 2]} \Phi(2 j, i-2 j)+\varphi \sum_{j=0}^{[i / 2]} \Phi(2 j, i-2 j), \quad i \geqslant 1 .
\end{aligned}
$$

Заметим, что из условия $u_{0}=0$ при $y_{2}=0$ следует, что $Z_{k}(y ; s)=a(s) r^{k} \sin (k \varphi)$, где $a \in C^{\infty}(\gamma)$. Далее, обозначим через $\mathscr{A}_{k}$ множество функций из $C^{\infty}(\bar{\Omega} \backslash \gamma)$, обрашаюшихся в нуль на $\Gamma \backslash \gamma$ и имеюших дифференцируемые асимптотики из $\widetilde{A}_{k}$.

Лемма 4.5. Для любого натурального $k$ и любъх $Z_{k}(y ; s)=a(s) r^{k} \sin (k \varphi)$, где $а \in C^{\infty}(\gamma)$, и $\widetilde{F} \in \widetilde{\mathscr{A}}_{k}$ существует асимптотическое $($ при $r \rightarrow 0)$ решение $\widetilde{U} \in \widetilde{\mathscr{A}}_{k}$ уравнения

$$
-\left(\Delta+\lambda_{0}\right) \widetilde{U}=\widetilde{F}
$$

имеющее главный член $Z_{k} r^{-2 k}$.

ДокАЗАТЕЛЬСтво леммы проводится по индукции. Для коэффициентов ряда (4.4) в силу (4.3) и предположения индукции получаются уравнения

$$
\begin{aligned}
\Delta_{y} u_{i}(y ; s)= & \sum_{j=0}^{i} \Phi(-2 k+2 j-4, k+i-2 j+2), \quad 1 \leqslant i \leqslant k \\
\Delta_{y} u_{k+i}(y ; s)= & \sum_{j=0}^{k} \Phi(-2 k+2 j-4,2 k+i-2 j+2)+\ln r \sum_{j=1}^{[i / 2]} \Phi(2 j-2, i-2 j) \\
& +\sum_{j=0}^{[i / 2]} \Phi(2 j-2, i-2 j)+\varphi \sum_{j=1}^{[i / 2]} \Phi(2 j-2, i-2 j), \quad i \geqslant 1 .
\end{aligned}
$$

Тогда в силу лемм $4.1,4.4$ существуют решения $\widetilde{u}_{t}$, удовлетворяющие уравнению (4.7) и имеющие структуру (4.5), (4.6). Заметим, что правая часть уравнения для $u_{k+1}$ не содержит $\ln r$, но содержит член $\Phi(-2,1)$. Поэтому из леммы 4.1 следует, что в решении $\widetilde{u}_{k+1}$ появится член $Z_{1} \ln r$. При $y_{2}=0, y \neq 0$, из определения $\Phi(i, j)$ следует, что

$$
\begin{aligned}
\widetilde{u}_{i}(y ; s) & = \begin{cases}\alpha_{i}^{(1)}(s) y_{1}-(k-i), & y_{1}>0, \\
\alpha_{i}^{(1)}(s)(-1)^{k-i}\left|y_{1}\right|^{-(k-i)}, & y_{1}<0,\end{cases} \\
\widetilde{u}_{k+i}(y ; s) & =\left\{\begin{array}{ll}
\alpha_{i}^{(2)}(s) y_{1}^{i}+\alpha_{i}^{(3)}(s) y_{1}^{i} \ln y_{1}, & y_{1}>0, \\
(-1)^{i}\left(\alpha_{i}^{(2)}(s)\left|y_{1}\right|^{i}+\alpha_{i}^{(3)}(s)\left|y_{1}\right|^{i} \ln \left|y_{1}\right|\right), & y_{1}<0,
\end{array} \quad i \geqslant 1 .\right.
\end{aligned}
$$


Тогда функции

$$
\begin{aligned}
u_{i} & =\widetilde{u}_{i}-\alpha_{i}^{(1)} r^{-(k-i)} \cos ((k-i) \varphi), & 1 \leqslant i \leqslant k, \\
u_{k+i} & =\widetilde{u}_{k+i}-\alpha_{i}^{(2)} r^{i} \cos (i \varphi)-\alpha_{i}^{(3)}\left(r^{i} \cos (i \varphi) \ln r-\varphi r^{i} \sin (i \varphi)\right), & i \geqslant 1,
\end{aligned}
$$

равны нулю при $y_{2}=0$ и удовлетворяют (4.5), (4.6). Лемма доказана.

ЗАмЕчАнИЕ 4.1 . При $k=1$ и $\widetilde{F}=0$ в формальном асимптотическом решении возникновение логарифмического члена происходит следующим образом:

$$
\widetilde{U}(y ; s)=a(s) r^{-1} \sin \varphi+\cdots+\widetilde{\mathscr{D}}(a(s)) y_{2} \ln r+\cdots,
$$

где $\widetilde{\mathscr{D}}=\alpha(s) \frac{d^{2}}{d s^{2}}+\beta(s), \alpha(s), \beta(s) \in C^{\infty}(\gamma)$ зависят от геометрии $\gamma$ и $\Omega($ см. (1.7)).

Лемма 4.6. Для любих $Z_{j}(y ; s)=a(s) r^{j} \sin (j \varphi)$, әде $a \in C^{\infty}(\gamma)$, u $F \in \mathscr{A}_{j}$ существует постоянная $\Lambda$ и существует функиия $U \in \mathscr{A}_{j}$, которая является решением (4.2), а главный член ее асимптотики при $r \rightarrow 0$ имеет вид $Z_{j} r^{-2 j}$.

ДокАЗАтЕльство. Пусть $\widetilde{F}$ - асимптотика $F$ при $r \rightarrow 0, \chi(t)$ - бесконечно дифференцируемая срезающая функция, тождественно равная единице при $t<1$ и нулю при $t>2$, а $R$ - достаточно малое фиксированное положительное число. Будем искать функцию $U(x)$ в виде

$$
U(x)=u_{N}(x)=\chi\left(r R^{-1}\right) \widetilde{U}_{N}(x)+\widetilde{u}_{N}(x),
$$

где ряд $\widetilde{U}$ удовлетворяет утверждению леммы $4.5, \widetilde{U}_{N}-$ его частичная сумма до членов $O\left(r^{N}\right)$ включительно, а $\widetilde{u}_{N}(x)$ - некоторая достаточно гладкая функция. Тогда для $\widetilde{u}_{N}(x)$ получаем краевую задачу

$$
\begin{gathered}
-\left(\Delta+\lambda_{0}\right) \widetilde{u}_{N}=\Lambda(N) \psi_{0}+G_{N}, \quad x \in \Omega, \quad \widetilde{u}_{N}=0, \quad x \in \Gamma, \\
G_{N}=\Delta \chi \widetilde{U}_{N}+2 \sum_{i=1}^{3} \frac{\partial \chi}{\partial x_{i}} \frac{\partial \widetilde{U}_{N}}{\partial x_{i}}+\chi\left(\Delta+\lambda_{0}\right) \widetilde{U}_{N}+F .
\end{gathered}
$$

Первые два слагаемых в сумме (4.9) принадлежат классу $C^{\infty}(\bar{\Omega})$, так как $\Delta \widetilde{U}_{N} \in$ $C^{\infty}(\bar{\Omega} \backslash \gamma)$, а носители функций $\Delta\left(\chi\left(r R^{-1}\right)\right), \frac{\partial \chi\left(r R^{-1}\right)}{\partial x_{i}}$ отделены от $\gamma$. Далее, в силу выражений для членов ряда $\widetilde{U}$ вытекает, что $\left(\Delta+\lambda_{0}\right) \widetilde{U}_{N}+F=O\left(r^{N-2} \ln r\right)$ при $r \rightarrow 0$. Следовательно, $G_{N} \in C^{N-3}(\bar{\Omega})$. Так как $\lambda_{0}-$ собственное значение предельной задачи, то равенство $\left(G_{N}+\Lambda(N) \psi_{0}, \psi_{0}\right)_{\Omega}=0$ является необходимым и достаточньм условием разрешимости $(4.8)$, т.е. $\Lambda(N)=-\left(G_{N}, \psi_{0}\right)_{\Omega}$. При таком выборе $\Lambda(N)$ краевая задача (4.8) разрешима, причем функция $\widetilde{u}_{N} \in C^{N-1}(\bar{\Omega})$. Разлагая функцию $\widetilde{u}_{N}$ по формуле Тейлора, получаем

$$
\widetilde{u}_{N}(x)=\sum_{j=1}^{N-2} Q_{j}(y ; s)+O\left(r^{N-1}\right), \quad r \rightarrow 0
$$


Покажем, что функцию $u_{N}(x)$ можно выбрать не зависяшей от $N$. Пусть $N_{1} \geqslant$ $N \geqslant 4$. Тогда очевидно, что $\widetilde{u}_{N_{1}}(x)-\widetilde{u}_{N}(x) \in C^{3}(\bar{\Omega})$. Функция $\chi\left(r R^{-1}\right)\left(\widetilde{U}_{N_{1}}(x)-\right.$ $\left.\widetilde{U}_{N}(x)\right)$ принадлежит классу $C^{N}(\bar{\Omega})$ в силу леммы 4.5. Таким образом, получили, что разность $u_{N, N_{1}}=u_{N_{1}}(x)-u_{N}(x) \in C^{3}(\bar{\Omega})$. Так как каждая из функций $u_{N_{1}}$ и $u_{N}$ удовлетворяет (4.2), то $u_{N, N_{1}}$ является решением этой краевой задачи с $\Lambda=\Lambda\left(N_{1}\right)-\Lambda(N)$ и $F=0$. Следовательно, $\Lambda\left(N_{1}\right)=\Lambda(N)$ и $u_{N, N_{1}}$ является собственной функцией. Заметим, что решение (4.8) определено с точностью до собственной функции $\psi_{0}$. Поэтому для определенности при $N \geqslant 4$ на $u_{N}$ наложим условие

$$
\int_{\Omega} u_{4, N} \psi_{0} d x=0
$$

Тогда получаем, что $u_{N}$ не зависит от $N$. Из произвола в выборе $N$ следует, что $U \in \mathscr{A}_{k}$. Таким образом, лемма 4.6 доказана.

ДокаЗАТЕЛЬСтво ЛЕммы 2.1. В рассматриваемом случае $F=0, Z_{1}(y ; s)=$ $a(s) r \sin \varphi$. Из леммы 4.6 следует, что существует функция $U=U^{(2)} \in \mathscr{A}_{1}$, которая является решением (2.12), а главный член ее асимптотики при $r \rightarrow 0$ имеет вид $Z_{1} r^{-2}$. Вычислим $\Lambda=\Lambda^{(2)}$. Умножим обе части равенства в $(2.12)$ на $\psi_{0}$, возьмем интеграл по $\Omega \backslash S_{\mu}^{2}$ от обеих частей. Интегрируя полученное равенство по частям, вьводим

$$
\Lambda^{(2)}=\int_{\Omega \cap \partial S_{\mu}^{2}} \psi_{0} \frac{\partial U^{(2)}}{\partial r} d S-\int_{\Omega \cap \partial S_{\mu}^{2}} \frac{\partial \psi_{0}}{\partial r} U^{(2)} d S .
$$

Подставляя в последнее равенство главные члены асимптотик $\psi_{0} \sim c_{01} r \sin \varphi$ и $U^{(2)} \sim a r^{-1} \sin \varphi$ при $r \rightarrow 0$ и переходя к пределу при $\mu \rightarrow 0$, получаем (2.13). Лемма 2.1 доказана.

ЗАмЕчАниЕ 4.2. В силу замечания 4.1 и доказательства леммы 4.6 легко видеть, что асимптотика построенной в лемме 2.1 функции имеет вид

$$
U^{(2)}(y ; s)=a(s) r^{-1} \sin \varphi+\cdots+\widetilde{\mathscr{D}}(a(s)) y_{2} \ln r+\cdots,
$$

где выделен первый появляюшийся логарифмический член.

Теперь рассмотрим случай, когда кривая $\gamma$ лежит в области $\Omega$ (т.е. задачу А2). Будем изучать сингулярные на $\gamma$ решения краевых задач

$$
-\left(\Delta+\lambda_{0}\right) U=F+\Lambda \psi_{0}, \quad x \in \Omega \backslash \gamma, \quad U=0, \quad x \in \Gamma,
$$

где $F$ - функция, имеющая особенность на $\gamma$.

При $k \geqslant 1$ обозначим через $\widetilde{\mathscr{B}}_{k}$ множество рядов вида (4.4)-(4.6), где последняя сумма в (4.6) отсутствует. Заметим, что на эти члены не налагается требование обращения в нуль при $y_{2}=0$. Пусть, далее, $\widetilde{\mathscr{B}}_{0}-$ множество рядов вида (4.4), где

$$
\begin{gathered}
u_{0}(y ; s)=g(s) \ln r, \\
u_{n}(y ; s)=\ln r \sum_{j=0}^{[n / 2]} \Phi(2 j, n-2 j)+\sum_{j=1}^{[n / 2]} \Phi(2 j, n-2 j) .
\end{gathered}
$$

Аналогично лемме (4.5) доказываются следующие утверждения. 
Лемма 4.7. Для любых $g(s) \in C^{\infty}(\gamma)$ существует асимптотическое ( $r \rightarrow 0$ ) решение $\widetilde{U} \in \widetilde{\mathscr{B}}_{0}$ уравнения (4.7) с $\widetilde{F} \in \widetilde{\mathscr{B}}_{0}$, имеющее главный член $g(s) \ln r$.

ЛЕмма 4.8. Для любого натурального $k$ и любъх $Z_{k}$ и $\widetilde{F} \in \widetilde{\mathscr{B}}_{k}$ существует асимптотическое $($ при $r \rightarrow 0)$ решение $\widetilde{U} \in \widetilde{\mathscr{B}}_{k}$ уравнения (4.7), имеющее главный член $Z_{k} r^{-2 k}$.

Обозначим через $\mathscr{B}_{k}$ множество функций из $C^{\infty}(\bar{\Omega} \backslash \gamma)$, обрашаюшихся в нуль на Г и имеющих дифференцируемые асимптотики из $\widetilde{\mathscr{B}}_{k}$, а через $\mathscr{B}_{k}^{(0)}$ при $k \geqslant 1-$ множество функций, представимых в виде $U^{(1)}+U^{(2)}$, где $U^{(1)} \in \mathscr{B}_{k}, U^{(2)} \in \mathscr{B}_{0}$. С учетом лемм $4.7,4.8$ аналогично лемме 4.6 доказываются следуюшие утверждения.

ЛЕмма 4.9. Для любъх $Z_{k}$ и $F \in \mathscr{B}_{k}^{(2)}$ существует постоянная $\Lambda$ и существует функция $U \in \mathscr{B}_{k}^{(2)}$, которая является решением (4.10), а главный член ее асимптотики при $r \rightarrow 0$ имеет вид $Z_{k} r^{-2 k}$.

Лемма 4.10. Для любых $g \in C^{\infty}(\gamma)$ и $F \in \mathscr{B}^{(0)}$ существует постоянная $\Lambda$ и существует функция $U \in \mathscr{B}^{(0)}$, которая является решением (4.10), а главный член ее асимптотики при $r \rightarrow 0$ имеет вид $g(s) \ln r$.

ДОКАЗАТЕЛЬСТво ЛЕМмы 3.2. С учетом лемм 4.9, 4.10 доказательство леммы 3.2 проводится интегрированием по частям совершенно аналогично доказательству леммы 2.1.

\section{§5. Внутренние разложения для краевых задач (1.2), (1.3)}

Обозначим $\mathbb{R}_{+, \xi}^{2}=\left\{\xi: \xi_{2}>0\right\}$. Рассмотрим краевую задачу

$$
\Delta_{\xi} V=F, \quad \xi \in \mathbb{R}_{+, \xi}^{2}, \quad V=0, \quad \xi \in \Gamma_{1}, \quad \frac{\partial V}{\partial \xi_{2}}=0, \quad \xi \in \gamma_{1}
$$

где $F$ растет при $\rho \rightarrow \infty$.

При $q \geqslant 1$ через $\widetilde{\mathscr{F}}_{q}$ обозначим множество рядов вида

$$
\widetilde{V}(\xi ; s)=\sum_{j=-q}^{\infty} H_{-j}(\xi ; s)+\ln \rho \sum_{j=1}^{q-2} Q_{j}(\xi ; s),
$$

где $H_{q}$ - однородные функции, представимые в виде $H_{q}(\xi ; s)=Q_{q+2 i}(\xi ; s) \rho^{-2 i}$ при некотором целом $i$, коэффициенты однородных полиномов $Q_{j}(\xi ; s)$ из $C^{\infty}(\gamma)$, а через $\mathscr{F}_{q}^{(0)}$ обозначим множество функций $v$ обладающих следуюшими свойствами:

k1) $v \in C^{\infty}\left(\left\{\xi: \xi_{2} \geqslant 0, \xi \neq\left(f_{1}(s), 0\right), \xi \neq\left(f_{2}(s), 0\right)\right\}\right)$;

k2) при $\rho \rightarrow \infty$ функция $v$ имеет дифференцируемые по $\xi$ и $s$ асимптотики из $\widetilde{\mathscr{F}}_{q}$, т.е. для любых целых неотрицательных $\alpha_{1}, \alpha_{2}, \alpha_{3}$ справедлива оценка

$$
\left|\frac{\partial^{\alpha_{1}+\alpha_{3}+\alpha_{3}}\left(v-V_{N}\right)}{\partial \xi_{1}^{\alpha_{1}} \partial \xi_{2}^{\alpha_{2}} \partial s^{\alpha_{3}}}\right|<C_{N, \alpha_{1}, \alpha_{2}, \alpha_{3}} \rho^{-N-1-\alpha_{1}-\alpha_{2}}
$$


где $V_{N}$ - частичная сумма членов в асимптотике $v$ на бесконечности до членов $O\left(\rho^{-N}\right)$ включительно;

$\mathrm{k} 3)$ для любого $R>0$ функция $\chi(\rho R) v(\xi ; s)$, рассматриваемая как отображение $\gamma$ в $L_{2}\left(\mathbb{R}_{+, \xi}^{2}\right)$, является бесконечно дифференцируемым по $s \in \gamma$.

Пусть, далее, $\eta=\left(\eta_{1}, \eta_{2}\right), \eta_{1}=\frac{2}{f_{2}-f_{1}}\left(\xi_{1}-\frac{f_{1}+f_{2}}{2}\right), \eta_{2}=\frac{2}{f_{2}-f_{1}} \xi_{2}$. Обозначим через $\mathscr{F}_{q}^{(1)}$ множество функций $v$, обладающих следующими свойствами:

11) справедливы свойства $\mathrm{k} 1$ ) и $\mathrm{k} 2)$;

12) функция $v(\xi ; s)=0$ на $\gamma_{1}$;

13) для любого $R>0$ функция $\chi(\rho(\eta) R) v(\xi(\eta) ; s)$, рассматриваемая как отображение $\gamma$ в $W_{2}^{1}\left(\mathbb{R}_{+, \eta}^{2}\right)$, является бесконечно дифференцируемой по $s \in \gamma$.

И наконец, обозначим через $\mathscr{F}_{q}^{(-1)}$ множество функций $v$, обладающих следующими свойствами:

$\mathrm{m} 1$ ) справедливы свойства $\mathrm{k} 1$ ) и $\mathrm{k} 2$ );

$\mathrm{m} 2)$ сушествуют $R$ такие, что функции $\chi(\rho(\eta) R) v(\xi(\eta) ; s)$ могут быть представлены в виде $g_{0}(\eta ; s)+\sum_{i=1}^{2} \frac{\partial g_{i}(\eta ; s)}{\partial \eta_{i}}$

m3) функция $g_{2}=0$ на $\widetilde{\gamma}_{1}$;

$\mathrm{m} 4$ ) функции $g_{i}$, рассматриваемые как отображение $\gamma$ в $L_{2}\left(\mathbb{R}_{+, \eta}^{2}\right)$, являются бесконечно дифференцируемьми по $s \in \gamma$.

ЗАмечАниЕ 5.1. Из определений $\mathscr{F}_{q}^{(1)}, \mathscr{F}_{q}^{(-1)}$ следует, что если $v \in \mathscr{F}_{q+2}^{(1)}$, то

$$
\begin{aligned}
& R_{i, 2}(\xi, s, D) v(\xi ; s) \in \mathscr{F}_{q}^{(-1)}, \\
& R_{i, 1}(\xi, s, D) v(\xi ; s) \in \mathscr{F}_{q+1}^{(-1)}, \\
& R_{i, 0}(\xi, s, D) v(\xi ; s) \in \mathscr{F}_{q+2}^{(-1)} .
\end{aligned}
$$

Лемма 5.1. Пусть $G \in \mathscr{F}_{q}^{(0)}$, а ряд $\widetilde{V} \in \widetilde{\mathscr{F}}_{q+2}$ является асимптотическим $($ при $\rho \rightarrow \infty)$ решением краевой задачи

$$
\Delta_{\xi} \tilde{V}=G, \quad \xi \in \mathbb{R}_{+, \xi}^{2}, \quad \widetilde{V}=0, \quad \xi_{2}=0
$$

Тогда существует решение $v \in \mathscr{F}_{q+2}^{(1)}$ краевой задачи

$$
\Delta_{\xi} v=G, \quad \xi \in \mathbb{R}_{+, \xi}^{2}, \quad v=0, \quad \xi \in \Gamma_{1}, \quad \frac{\partial v}{\partial \xi_{2}}=0, \quad \xi \in \gamma_{1}
$$

имеющее при $\rho \rightarrow \infty$ бесконечно дифференцируемье асимптотики

$$
v(\xi ; s)=\tilde{V}(\xi ; s)+\sum_{i=1}^{\infty} a_{i}(s) \rho^{-i} \sin (i \varphi)
$$


Доказательство. Решение краевой задачи (5.3) при достаточно малых $R$ (а именно таких, что $R^{-1}>f_{2}-f_{1}$ ) будем искать в виде

$$
v(\xi ; s)=v_{N}(\xi ; s)=(1-\chi(\rho R)) \widetilde{V}_{N}(\xi ; s)+w_{N}^{(0)}(\xi ; s),
$$

где $\widetilde{V}_{N}$ - частичная сумма формального асимптотического решения $\widetilde{V}$. Тогда для $w_{N}^{(0)}$ получаем краевую задачу

$$
\Delta_{\xi} w_{N}^{(0)}=\widetilde{G}_{N}, \quad \xi \in \mathbb{R}_{+, \xi}^{2}, \quad w_{N}^{(0)}=0, \quad \xi \in \Gamma_{1}, \quad \frac{\partial w_{N}^{(0)}}{\partial \xi_{2}}=0, \quad \xi \in \gamma_{1} .
$$

Так как $G \in \mathscr{F}_{q}^{(0)}$, то легко видеть, что в силу свойства $\left.\mathrm{k} 2\right)$ функция $\widetilde{G}_{N}$ имеет при $\rho \rightarrow \infty$ бесконечно дифференцируемую по $s$ и $\xi$ асимптотику $\widetilde{G}_{N}=O\left(\rho^{-N-1}\right)$, а в силу свойства $\mathrm{k} 3)$ функция $\widetilde{G}_{N}$, рассматриваемая как отображение $\gamma$ в $L_{2}\left(\mathbb{R}_{+, \xi}^{2}\right)$, является бесконечно дифференцируемой по $s \in \gamma$. В свою очередь, будем искать решение краевой задачи (5.6) в виде $w_{N}^{(0)}=w_{N}^{(1)}+w_{N}^{(2)}$, где

$$
\begin{gathered}
\Delta_{\xi} w_{N}^{(1)}=\widetilde{G}_{N}, \quad \xi \in \mathbb{R}_{+, \xi}^{2}, \quad w_{N}^{(1)}=0, \quad \xi_{2}=0 \\
\Delta_{\xi} w_{N}^{(2)}=0, \quad \xi \in \mathbb{R}_{+, \xi}^{2}, \quad w_{N}^{(2)}=0, \quad \xi \in \Gamma_{1}, \quad \frac{\partial w_{N}^{(2)}}{\partial \xi_{2}}=-\frac{\partial w_{N}^{(1)}}{\partial \xi_{2}}, \quad \xi \in \gamma_{1} .
\end{gathered}
$$

Из явной формулы решения задачи Дирихле в полуплоскости, упомянутых выше свойств $\widetilde{G}_{N}$ и теоремы Лебега следует существование функции $w_{N}^{(1)} \in W_{2}^{2}\left(\mathbb{R}_{+, \xi}^{2}\right)$, обладающей свойством 13) и имеющей асимптотику

$$
w_{N}^{(k)}=\sum_{i=1}^{N-2} \widetilde{a}_{i}(s) \rho^{-i} \sin (i \varphi)+O\left(\rho^{-N+1}\right), \quad \rho \rightarrow \infty
$$

$(k=1)$. В свою очередь, из формулы Келдыша-Седова (см., например, [42]) следует существование функции $w_{N}^{(2)}$, обладающей свойством 13$)$, асимптотика которой при $\rho \rightarrow \infty$ имеет вид $(5.7)(k=2)$. Тогда функция $w_{N}^{(0)}$ также обладает свойством 13) и при $\rho \rightarrow \infty$ имеет асимптотику $(5.7)(k=0)$.

Покажем, что функция $v_{N}$ не зависит от $N$. Пусть $N_{1} \geqslant N \geqslant 4, v^{\left(N, N_{1}\right)}=$ $v_{N}-v_{N_{1}}$. Тогда для $v^{\left(N, N_{1}\right)}$ получаем краевую задачу

$$
\Delta_{\xi} v^{\left(N, N_{1}\right)}=0, \quad \xi \in \mathbb{R}_{+, \xi}^{2}, \quad v^{\left(N, N_{1}\right)}=0, \quad \xi \in \Gamma_{1}, \quad \frac{\partial v^{\left(N, N_{1}\right)}}{\partial \xi_{2}}=0, \quad \xi \in \gamma_{1} .
$$

Функция $v^{\left(N, N_{1}\right)}$ убывает при $\rho \rightarrow \infty$. Тогда в силу единственности решения этой краевой задачи функция $v^{\left(N, N_{1}\right)} \equiv 0$ при $\xi_{2} \geqslant 0$ (см., например, [42]) и, значит, $v_{N}$ не зависит от $N$. Из произвола в выборе $N$, формул (5.5) и (5.7) (при $k=0$ ) и теорем о повышении гладкости следует, что $v \in \mathscr{F}_{q+2}^{(1)}$. Лемма доказана. 
В переменных $\eta$ рассмотрим следуюшую краевую задачу:

$$
\left(\Delta_{\eta}-1\right) \widetilde{\phi}=-g_{0}+\sum_{i=1}^{2} \frac{\partial g_{i}}{\partial \eta_{i}}, \quad \eta \in \mathbb{R}_{+, \eta}^{2}, \quad \widetilde{\phi}=0, \quad \eta \in \widetilde{\Gamma}_{1}, \quad \frac{\partial \widetilde{\phi}}{\partial \eta_{2}}=g_{2}, \quad \eta \in \widetilde{\gamma}_{1},
$$

где $\widetilde{\Gamma}_{1}=\left\{\eta:\left|\eta_{1}\right|>1, \eta_{2}=0\right\}, \widetilde{\gamma}_{1}=\left\{\eta:\left|\eta_{1}\right|<1, \eta_{2}=0\right\}$. Пусть $W_{2,0}^{1}\left(\mathbb{R}_{+, \eta}^{2} ; \widetilde{\Gamma}_{1}\right)-$ замыкание в норме $W_{2}^{1}\left(\mathbb{R}_{+, \eta}^{2}\right)$ функций из $C^{\infty}\left(\left\{\eta: \eta_{2} \geqslant 0\right\}\right)$, обращающихся в нуль в окрестности $\widetilde{\Gamma}_{1}$. Пусть $g_{i}(\eta) \in L_{2}\left(\mathbb{R}_{+, \eta}^{2}\right)$. Решение краевой задачи (5.8) будем рассматривать в смысле обобшенных функций (см. [43]-[45]). То есть под решением будем понимать элемент $\widetilde{\phi} \in W_{2,0}^{1}\left(\mathbb{R}_{+, \eta}^{2} ; \widetilde{\Gamma}_{1}\right)$, удовлетворяющий интегральному равенству

$$
\left(\nabla_{\eta} \widetilde{\phi}, \nabla_{\eta} w\right)_{\mathbb{R}_{+, \eta}^{2}}+(\widetilde{\phi}, w)_{\mathbb{R}_{+, \eta}^{2}}=\left(g_{0}, w\right)_{\mathbb{R}_{+, \eta}^{2}}+\sum_{i=1}^{2}\left(g_{i}, \frac{\partial w}{\partial \eta_{i}}\right)_{\mathbb{R}_{+, \eta}^{2}}
$$

при любом $w \in W_{2,0}^{1}\left(\mathbb{R}_{+, \eta}^{2} ; \widetilde{\Gamma}_{1}\right)$. При любых фиксированных $g_{i}$ правая часть (5.9) есть линейньй ограниченный функционал, заданный на $W_{2,0}^{1}\left(\mathbb{R}_{+, \eta}^{2} ; \widetilde{\Gamma}_{1}\right)$. Поэтому из теоремы Рисса следует сушествование и единственность решения краевой задачи (5.8). Кроме того, если $g_{i} \in C^{\infty}\left(\left\{\eta: \eta_{2} \geqslant 0, \eta \neq(-1,0), \eta \neq(1,0)\right\}\right)$ и $g_{2}=0$ на $\widetilde{\gamma}_{1}$, то из теорем о повышении гладкости решений эллиптических краевых задач следует, что $\widetilde{\phi} \in C^{\infty}\left(\left\{\eta: \eta_{2} \geqslant 0, \eta \neq(-1,0), \eta \neq(1,0)\right\}\right)$ удовлетворяет однородному граничному условию Неймана на $\widetilde{\gamma}_{1}$ в обычном (классическом) смысле. Таким образом, подставляя $w=\widetilde{\phi}$ в $(5.9)$, получаем справедливость следуюшего утверждения.

Лемма 5.2. Пусть $g_{i}$ обладают свойствами $\left.\left.\mathrm{m} 3\right), \mathrm{m} 4\right)$. Тогда существует решение $\widetilde{\phi} \in W_{2}^{1}\left(\mathbb{R}_{+, \eta}^{2}\right)$ краевой задачи (5.8) с однородными граничными условиями. Функция $\widetilde{\phi}$, рассматриваемая как отображение $\gamma$ в $W_{2}^{1}\left(\mathbb{R}_{+, \eta}^{2}\right)$, является бесконечно дифференцируемой по $s \in \gamma$.

Лемма 5.3. Пусть $F \in \mathscr{F}_{q}^{(-1)}$, а ряд $\widetilde{V} \in \widetilde{\mathscr{F}}_{q+2}$ является формальныцм асимптотическим решением (при $\rho \rightarrow \infty)$ краевой задачи (5.2) с $G=F$. Тогда существует решение $V \in \mathscr{F}_{q+2}^{(1)}$ краевой задачи (5.1), имеющее при $\rho \rightarrow \infty$ дифференцируемую асимптотику (5.4).

ДокАЗАТЕЛЬство. При достаточно малых $R$ рассмотрим краевую задачу

$$
\left(\Delta_{\xi}-1\right) \phi=\chi F, \quad \xi_{2}>0, \quad \phi=0, \quad \xi \in \Gamma_{1}, \quad \frac{\partial \phi}{\partial \xi_{2}}=0, \quad \xi \in \gamma_{1} .
$$

Так как функция $F \in \mathscr{F}_{q}^{(-1)}$, то, переписывая краевую задачу (5.10) в переменных $\eta$, с учетом свойства т2) получаем краевую задачу $(5.8)$ для $\widetilde{\phi}(\eta ; s)=$ $\phi(\xi(\eta) ; s)$. Из леммы 5.2 следует сушествование решения $\widetilde{\phi}$, удовлетворяюшего свойству 12$)$ и однородному граничному условию Неймана на $\widetilde{\gamma}_{1}$. Переписывая $\widetilde{\phi}$ в переменных $\xi$, получаем, что сушествует решение $\phi(\xi ; s)=\widetilde{\phi}(\eta ; s)$ краевой задачи (5.10), обладающее свойствами 12$), 13)$. 
При достаточно малых $R>0$ будем искать решение $V$ в виде

$$
V(\xi ; s)=\chi(\rho R) \phi(\xi ; s)+\widetilde{v}(\xi ; s) .
$$

Тогда для $\widetilde{v}$ получаем краевую задачу

$$
\Delta_{\xi} \widetilde{v}=\widetilde{F}, \quad \xi_{2}>0, \quad \widetilde{v}=0, \quad \xi \in \Gamma_{1}, \quad \frac{\partial \widetilde{v}}{\partial \xi_{2}}=0, \quad \xi \in \gamma_{1},
$$

где $\widetilde{F} \in \mathscr{F}_{q}^{(0)}$. Так как $\widetilde{F}$ удовлетворяет условиям леммы 5.1 , то сушествует решение $\widetilde{v} \in \mathscr{F}_{q+2}^{(1)}$ краевой задачи (5.12), имеющее при $\rho \rightarrow \infty$ дифференцируемую асимптотику (5.4).

Из теорем о повышении гладкости, (5.11) и лемм 5.1 и 5.2 следует принадлежность $V \in \mathscr{F}_{q+2}^{(1)}$.

Теперь рассмотрим краевую задачу

$$
\begin{array}{rlrl}
\Delta_{\xi} V & =F(\xi ; s), & & \xi \in \mathbb{R}^{2} \backslash \bar{\omega} \\
\frac{\partial V}{\partial \nu} & =h\left(l_{\xi}, s\right), \quad & \xi \in \partial \omega
\end{array}
$$

где $F$ растет при $\rho \rightarrow \infty, h\left(l_{\xi}, s\right) \in C^{\infty}(\partial \omega \times \gamma)$.

При $q \geqslant 1$ обозначим через $\widetilde{\mathscr{G}}_{q}$ множество рядов вида

$$
\widetilde{V}(\xi ; s)=\sum_{j=-q}^{\infty} H_{-j}(\xi ; s)+\ln \rho \sum_{j=0}^{q-2} Q_{j}(\xi ; s),
$$

где $H_{q}$ - однородные функции, представимые в виде $H_{q}(\xi ; s)=Q_{q+2 i}(\xi ; s) \rho^{-2 i}$ при некотором целом $i$, коэффициенты однородных полиномов $Q_{j}(\xi ; s)$ из $C^{\infty}(\gamma)$. Пусть $\mathscr{G}_{q}$-множество функций $v$, обладающих следуюшими свойствами:

n1) $v \in C^{\infty}\left(\mathbb{R}^{2} \backslash \omega\right)$;

n2) $v$ имеет при $\rho \rightarrow \infty$ дифференцируемые по $\xi$ и $s$ асимптотики из $\widetilde{\mathscr{G}}_{q}$, т.е. для любых целых неотрицательных $\alpha_{1}, \alpha_{2}, \alpha_{3}$ справедлива оценка

$$
\left|\frac{\partial^{\alpha_{1}+\alpha_{3}+\alpha_{3}}\left(v-V_{N}\right)}{\partial \xi_{1}^{\alpha_{1}} \partial \xi_{2}^{\alpha_{2}} \partial s^{\alpha_{3}}}\right|<C_{N, \alpha_{1}, \alpha_{2}, \alpha_{3}} \rho^{-N-1-\alpha_{1}-\alpha_{2}}
$$

где $V_{N}$ - частичная сумма членов в асимптотике $v$ на бесконечности до членов $O\left(\rho^{-N}\right)$ включительно.

ЗАмечАниЕ 5.2. Из определения $\mathscr{G}_{q}$ следует, что если функция $v \in \mathscr{G}_{q+2}$, то

$$
\begin{aligned}
& R_{i, 2}(\xi, s, D) v(\xi ; s) \in \mathscr{G}_{q}, \\
& R_{i, 1}(\xi, s, D) v(\xi ; s) \in \mathscr{G}_{q+1}, \\
& R_{i, 0}(\xi, s, D) v(\xi ; s) \in \mathscr{G}_{q+2} .
\end{aligned}
$$


Лемма 5.4. Пусть $F \in \mathscr{G}_{j}$, а ряд $\widetilde{V} \in \widetilde{\mathscr{G}}_{j+2}$ является асимптотическим (при $\rho \rightarrow \infty)$ решением уравнения (5.13). Тогда существует решение $V \in \mathscr{G}_{j+2}$ краевой задачи (5.13), (5.14), имеющее при $\rho \rightarrow \infty$ дифференцируемье асимптотики

$$
V(\xi ; s)=\widetilde{V}(\xi ; s)+g(s) \ln \rho+\sum_{i=0}^{\infty} Z_{i}(\xi ; s) \rho^{-2 i} .
$$

ДоказАтельство. Решение краевой задачи (5.13), (5.14) при достаточно малых $R$ будем искать в виде

$$
V(\xi ; s)=v_{N}(\xi ; s)=(1-\chi(\rho R)) \widetilde{V}_{N}(\xi ; s)+w_{N}(\xi ; s),
$$

где $\widetilde{V}_{N}$ - частичная сумма формального асимптотического решения $\widetilde{V}$. Тогда для $w_{N}$ получаем краевую задачу

$$
\Delta_{\xi} w_{N}=G_{N}, \quad \xi \in \mathbb{R}^{2} \backslash \bar{\omega}, \quad \frac{\partial w_{N}}{\partial \nu}=h\left(l_{\xi}, s\right), \quad \xi \in \partial \omega
$$

где

$$
G_{N}=\Delta_{\xi} \widetilde{V}_{N}+2 \sum_{i=1}^{2} \frac{\partial \chi}{\partial \xi_{i}} \frac{\partial \widetilde{V}_{N}}{\partial \xi_{i}}+F_{N+1}
$$

и, следовательно, имеет при $\rho \rightarrow \infty$ бесконечно дифференцируемую по $s$ и $\xi$ асимптотику $G_{N}=O\left(\rho^{-N-1}\right)$. Так как $\partial \omega \in C^{\infty}, G_{N} \in C^{N-2}\left(\overline{\mathbb{R}^{2}} \backslash \omega\right)$, то функцию $G_{N}$ можно гладко продолжить в $\omega$. Тогда $G_{N} \in C^{N-2}\left(\mathbb{R}^{2}\right)$. Будем искать решение краевой задачи (5.16) в виде $w_{N}=w_{N}^{(1)}+w_{N}^{(2)}$, где

$$
\begin{gathered}
\Delta_{\xi} w_{N}^{(1)}=G_{N}, \quad \xi \in \mathbb{R}^{2}, \\
\Delta_{\xi} w_{N}^{(2)}=0, \quad \xi \in \mathbb{R}^{2} \backslash \bar{\omega}, \quad \frac{\partial w_{N}^{(2)}}{\partial \nu}=h\left(l_{\xi}, s\right)-\frac{\partial w_{N}^{(1)}}{\partial \nu}, \quad \xi \in \partial \omega .
\end{gathered}
$$

Так как $N \geqslant 4, G_{N} \in C^{N-2}\left(\mathbb{R}^{2}\right)$ и $G_{N}=O\left(\rho^{-N-1}\right)$ при $\rho \rightarrow \infty$, то, представляя решение $w_{N}^{(1)}$ в виде логарифмического потенциала, получаем, что $w_{N}^{(1)} \in$ $C^{N-2}\left(\mathbb{R}^{2}\right)$,

$$
V(\xi ; s)=\sum_{j=1}^{N-3} Z_{j}(\xi ; s) \rho^{-2 j}+O\left(\rho^{-N+2}\right), \quad \rho \rightarrow \infty .
$$

Представляя решение $w_{N}^{(2)}$ в виде потенциала простого слоя (см., например, [41]), получаем, что $w_{N}^{(2)} \in C^{\infty}\left(\mathbb{R}^{2} \backslash \omega\right)$,

$$
w_{N}^{(2)}(\xi ; s)=g(s) \ln \rho+\sum_{j=1}^{\infty} Z_{j}(\xi ; s) \rho^{-2 j}, \quad \rho \rightarrow \infty .
$$

Из формулы (5.15) следует, что краевая задача (5.13), (5.14) имеет решение $v_{N} \in$ $C^{N-2}\left(\mathbb{R}^{2} \backslash \omega\right)$. Аналогично тому, как это было сделано в лемме 5.1, доказывается независимость $v_{N}$ от $N$. Учитьвая, что $F$ обладает свойством n2), а также явные формулы для $w_{N}^{(1)}, w_{N}^{(2)}$, получаем, что $V \in \mathscr{G}_{j+2}$. 


\section{§6. Доказательство теоремы 1.1}

Будем строить асимптотику $\lambda_{\varepsilon}$ в виде (1.4), а асимптотики $\psi_{\varepsilon}$ в виде (1.8) при $x \in \Omega \backslash S_{\varepsilon^{1 / 2}}^{2}$ и в виде (1.9) при $x \in \Omega \cap S_{2 \varepsilon^{1 / 2}}^{2}$. Подставляя (1.4), (1.8), (1.9), (4.1) в (1.2), получаем краевые задачи для коэффициентов рядов (1.8), (1.9):

$$
\begin{gathered}
-\left(\Delta+\lambda_{0}\right) \psi_{2+k, q}=\lambda_{2+k, q} \psi_{0}+\sum_{i=0}^{k-2} \sum_{j=0}^{q} \lambda_{k-i, q-j} \psi_{2+i, j} \quad \text { в } \Omega, \\
\psi_{2+k, q}=0 \text { на } \Gamma \backslash \gamma ; \\
\Delta_{\xi} v_{1+k, q}=-\lambda_{0} v_{k-1, q}-\sum_{i=0}^{k-4} \sum_{j=0}^{q} \lambda_{k-i-2, q-j} v_{1+i, j} \\
+\sum_{i=1}^{k-2 q} R_{i, 2}(\xi, s, D) v_{k-i+1, q}+\sum_{i=0}^{k-2 q-1} R_{i, 1}(\xi, s, D) v_{k-i, q} \\
+\sum_{i=0}^{k-2 q-2} R_{i, 0}(\xi, s, D) v_{k-i-1, q} \text { при } \xi_{2}>0, \\
v_{1+k, q}=0 \text { на } \Gamma_{1}, \quad \frac{\partial v_{1+k, q}}{\partial \xi_{2}}=0 \text { на } \gamma_{1} .
\end{gathered}
$$

Здесь и далее будем считать равными нулю $\lambda_{k, q}, \psi_{k, q}, v_{k, q}$, индексы у которых не являются членами рядов $(1.4),(1.8),(1.9)$. На суммах $U(x, \varepsilon)$ вида (1.8) определим операторы $\mathscr{K}_{q, m}, \mathscr{K}_{q}$ и $K_{N}$ следующим образом. Коэффициенты ряда $U(x, \varepsilon)$ разложим в ряды при $r \rightarrow 0$ и перейдем к переменным $\xi$. В полученных рядах оставим только члены вида $\varepsilon^{q} \ln ^{m} \varepsilon \Phi(\xi)$. Этот ряд обозначим $\mathscr{K}_{q, m}(U(x, \varepsilon))$ и положим $K_{N}=\sum_{q \leqslant N} \sum_{m} \mathscr{K}_{q, m}$ ( $N$ может быть равно и бесконечности).

Обозначим через $\Psi_{\varepsilon}(x)$ ряд вида (1.8). Из определения $K_{N}, \mathscr{A}_{i}$ и $\widetilde{\mathscr{F}}_{q}$ вытекает справедливость следующего утверждения.

Лемма 6.1. Пусть $\psi_{2+k, q} \in \mathscr{A}_{k+1-2 q}$. Тогда

$$
\begin{gathered}
K_{\infty}\left(\Psi_{\varepsilon}(x)\right)=\sum_{i=1}^{\infty} \sum_{j=0}^{[(i-1) / 2]} \varepsilon^{i} \ln ^{j} \varepsilon V_{i, j}(\xi ; s), \\
V_{i, j} \in \widetilde{\mathscr{F}}_{i-2 j}, \quad V_{i, j}\left(\xi_{1}, 0 ; s\right)=0, \quad \xi_{1} \neq 0 .
\end{gathered}
$$

Если к тому же $\psi_{2+k, q}-$ решения (6.1), то ряды $V_{i, j}$ являются формальными асимптотическими решениями уравнений (6.2) при $\rho \rightarrow \infty$, где в правой части функиии $v_{i, j}$ заменены на ряды $V_{i, j}$.

Будем искать $\psi_{2+k, q}, \lambda_{2+k, q}$ соответственно в виде

$$
\psi_{2+k, q}(x)=\sum_{j=2 q}^{k} \Psi_{2+k, q}^{(j)}, \quad \lambda_{2+k, q}(x)=\sum_{j=2 q}^{k} \Lambda_{2+k, q}^{(j)} .
$$

Далее будем считать равными нулю $\Psi_{k, q}^{(m)}, \Lambda_{k, q}^{(n)}$, индексы у которых не являются членами (6.4). 
Лемма 6.2. Если $\Psi_{2+k, q}^{(t)} \in \mathscr{A}_{k+1-t}$, то функиия $\psi_{2+k, q}$, определенная равенством (6.4), принадлежсит $\mathscr{A}_{k+1-2 q}$. Если к тому жсе $\Psi_{2+k, q}^{(t)}$ являются решениями краевых задач

$$
\begin{gathered}
-\left(\Delta+\lambda_{0}\right) \Psi_{2+k, q}^{(t)}=\Lambda_{2+k, q}^{(t)} \psi_{0}+\sum_{i=0}^{k-2} \sum_{j=0}^{q} \sum_{m+n=t} \Lambda_{k-i, q-j}^{(m)} \Psi_{2+i, j}^{(n)} \quad \text { npu } x \in \Omega, \\
\Psi_{2+k, q}^{(t)}=0 \quad \text { npu } \quad x \in \Gamma \backslash \gamma,
\end{gathered}
$$

то $\psi_{2+k, q}$ являются решениями краевых задач (6.1).

ДоказАтельство. Так как $\mathscr{A}_{q} \subset \mathscr{A}_{q+1}$, то из (6.4) следует первое утверждение. Суммируя (6.5) по $t$ от $2 q$ до $k$, получаем справедливость второго утверждения леммы.

Обозначим

$$
\begin{aligned}
\psi^{(N)}(x, \varepsilon) & =\psi_{0}(x)+\sum_{i=0}^{\infty} \sum_{j=0}^{\left[\frac{1}{2} \min \{N, i\}\right]} \varepsilon^{2+i} \ln ^{j} \varepsilon \psi_{2+i, j}^{(N)}(x), \\
\psi^{(-1)}(x, \varepsilon) & =\psi_{0}(x), \quad \psi_{2+k, j}^{(N)}(x)=\sum_{t=2 j}^{\min \{N, k\}} \Psi_{2+k, j}^{(t)}(x) .
\end{aligned}
$$

ЗАмечаниЕ 6.1. Так как $\Psi_{2+k, q}^{(j)} \in \mathscr{A}_{k+1-j}$, то из определений $K_{N}, \psi^{(N)}(x, \varepsilon)$ следует, что $K_{N}\left(\psi^{(N-1)}\right)=K_{N}\left(\Psi_{\varepsilon}\right)$.

Из лемм 6.1 и 6.2 вытекает справедливость следующего утверждения.

Лемма 6.3. Пусть $\Psi_{2+k, q}^{(j)} \in \mathscr{A}_{k+1-j}$. Тогда рядъь $V_{i, j}$ в (6.3) имеют вид

$$
V_{i, j}(\xi ; s)=\widetilde{V}_{i, j}(\xi ; s)+\sum_{t=1}^{\infty} a_{t}^{(i, j)}(s) \rho^{-t} \sin (t \varphi)
$$

$a_{t}^{(i, j)}(s) \rho^{-t} \sin (t \varphi)-$ главный член асимптотики $\Psi_{t+i, j}^{(i-1)}$ в нуле,

$$
\widetilde{V}_{i, j}(\xi ; s)=\varepsilon^{-i} \ln ^{-j} \mathscr{K}_{i, j}\left(\psi^{(i-2)}(x, \varepsilon)\right), \quad \widetilde{V}_{i, j} \in \widetilde{\mathscr{F}}_{i-2 j},
$$

и, в частности,

$$
\widetilde{V}_{1,0}(\xi ; s)=c_{01}(s) \xi_{2}, \quad \widetilde{V}_{2 t+1, t}(\xi ; s)=d_{t}(s) \xi_{2}, \quad t>0
$$

где $d_{t}(s) y_{2} \ln r-$ первъй логарифмический член в асимптотике $\psi_{2 t, t-1}(x)=$ $\Psi_{2 t, t-1}^{(2 t-2)}(x)$ на $\gamma$.

Если к тому же $\Psi_{2+k, q}^{(t)}$ являются решениями (6.5), то ряды $\widetilde{V}_{1+k, q}$ являются формальными асимптотическими решениями (6.2) при $\rho \rightarrow \infty$, әде в правой части функичи $v_{1+i, j}$ заменены на $V_{1+i, j}$.

Обозначим через $\widehat{v}_{N}(\xi, s, \varepsilon)$ частичную сумму ряда (1.9). 
ТеОрема 6.1. Существуют рядьи (1.4), (1.8) и (1.9) такие, что

1) выполняются равенства (1.5), (1.6);

2) функиии $\psi_{2+i, q} \in \mathscr{A}_{i+1-2 q}$ являются решениями (6.1);

3) функиии $v_{i, q} \in \mathscr{F}_{i-2 q}^{(1)}$ являются решениями (6.2);

4) для любого $N>0$ выполняется равенство

$$
K_{N}\left(\Psi_{\varepsilon}(x)\right)=\widehat{v}_{N}(\xi, s, \varepsilon), \quad \rho \rightarrow \infty
$$

ДокАЗАтельство. Ранее было доказано, что существует решение $v_{1,0}=v_{1}$ краевой задачи (6.2), определенное равенством (2.7), с асимптотикой

$$
v_{1,0}(\xi ; s)=\widetilde{V}_{1,0}(\xi ; s)+\sum_{i=1}^{\infty} \beta_{i}(s) \rho^{-i} \sin (i \varphi), \quad \rho \rightarrow \infty
$$

Из определения $\mathscr{F}_{q}^{(1)}$ и $v_{1,0}$ следует, что $v_{1,0} \in \mathscr{F}_{1}^{(1)}$.

Выберем главные члены $a_{i}^{(1,0)}(s) r^{-i} \sin (i \varphi)$ асимптотик $\Psi_{i+1,0}^{(0)}(x)$ равными $\beta_{i}(s) r^{-i} \sin (i \varphi)$ из (6.7). Тогда из (6.7) и леммы 6.3 следует, что

$$
v_{1,0}(\xi ; s)=V_{1,0}(\xi ; s), \quad \rho \rightarrow \infty
$$

С другой стороны, в силу леммы 4.6 существуют постоянные $\Lambda_{i+1,0}^{(0)}$ и функции $\Psi_{i+1,0}^{(0)} \in \mathscr{A}_{i}$ с заданньми главными членами асимптотик такие, что (6.5) выполняется при $t=0$. Таким образом, построен ряд $\psi^{(0)}(x, \varepsilon)$. При этом в силу леммы 2.1 имеем $\Psi_{2,0}^{(0)}=U^{(2)}\left(x ; \beta_{1}(s)\right)$, а потому справедливо $(1.5)$ для $\Lambda_{2,0}^{(0)}=\lambda_{2,0}$. Так как $K_{1}\left(\psi^{(0)}\right)=K_{1}\left(\Psi_{\varepsilon}\right)$ (замечание 6.1), то (6.6) вьполняется для $N=1$.

Дальнейшее доказательство проводится по индукции. Пусть существует ряд $\psi^{(N-2)}(x, \varepsilon)$, коэффициенты которого являются решением краевых задач (6.5), функции $v_{i, j}$ при $i \leqslant N-1$ и $j \leqslant[(i-1) / 2]$ являются решениями краевых задач $(6.2)$ и такие, что выполняется равенство

$$
K_{N-1}\left(\psi^{(N-2)}\right)=\widehat{v}_{N-1}
$$

Так как $\widetilde{V}_{N, q} \in \widetilde{\mathscr{F}}_{N-2 q}$, то в силу замечания 5.1 и леммы 5.3 сушествует решение $v_{N, q} \in \mathscr{F}_{N-2 q}^{(1)}$ краевой задачи $(6.2)$ с асимптотикой

$$
v_{N, q}(\xi ; s)=\widetilde{V}_{N, q}(\xi ; s)+\sum_{i=1}^{\infty} a_{i}(s) \rho^{-i} \sin (i \varphi), \quad \rho \rightarrow \infty
$$

Выберем главные члены $a_{i}^{(N, q)}(s) r^{-i} \sin (i \varphi)$ асимптотик $\Psi_{i+N, q}^{(N-1)}(x)$ равными $a_{i}(s) r^{-i} \sin (i \varphi)$. Тогда из леммы 6.3 следует, что

$$
v_{N, q}(\xi ; s)=V_{N, q}(\xi ; s), \quad \rho \rightarrow \infty \text {. }
$$


С другой стороны, в силу леммы 4.6 существуют постоянные $\Lambda_{i+N, q}^{(N-1)}$ и функции $\Psi_{i+N, q}^{(N-1)} \in \mathscr{A}_{i}$ с заданными главными членами асимптотик такие, что (6.5) выполняется при $t=N-1$. Таким образом, существует ряд $\psi^{(N-1)}(x, \varepsilon)$, коэффициенты которого являются решением краевых задач (6.5), функции $v_{i, j}$ при $i \leqslant N$ и $j \leqslant[(i-1) / 2]$ являются решениями краевых задач (6.2), причем в силу (6.8), (6.9) справедливо равенство $K_{N}\left(\psi^{(N-1)}\right)=\widehat{v}_{N}$. Из замечания 6.1 и произвола в выбоpe $N$ вытекает справедливость (6.6) для любого $N$.

Осталось показать справедливость (1.6).

Краевые задачи (6.1) и (6.2) для $\psi_{2 t, t-1}=\Psi_{2 t, t-1}^{(2 t-2)}, \lambda_{2 t, t-1}=\Lambda_{2 t, t-1}^{(2 t-2)}$ и $v_{1+2 t, t}$ имеют вид

$$
\begin{gathered}
-\Delta \psi_{2 t, t-1}=\lambda_{0} \psi_{2 t, t-1}+\lambda_{2 t, t-1} \psi_{0}, \quad x \in \Omega, \quad \psi_{2 t, t-1}=0, \quad x \in \Gamma \backslash \gamma \\
\Delta_{\xi} v_{2 t+1, t}=0, \quad \xi_{2}>0, \quad v_{2 t+1, t}=0, \quad \xi \in \Gamma_{1}, \quad \frac{\partial v_{2 t+1, t}}{\partial \xi_{2}}=0, \quad \xi \in \gamma_{1} .
\end{gathered}
$$

С другой стороны, в силу замечания 4.2

$$
\psi_{2 t, t-1}(x)=a_{t}(s) r^{-1} \sin \varphi+\cdots+d_{t}(s) y_{2} \ln r+\cdots, \quad d_{t}(s)=\widetilde{\mathscr{D}}\left(a_{t}(s)\right),
$$

причем из (2.10) и (2.8) имеем

$$
a_{1}(s)=\frac{c_{01}(s)}{8}\left(f_{2}(s)-f_{1}(s)\right)^{2} .
$$

И наконец, в силу леммы 6.3

$$
\widetilde{V}_{1+2 t, t}(\xi ; s)=d_{t}(s) \xi_{2}, \quad t>0 .
$$

Следовательно,

$$
v_{1+2 t, t}=d_{t} \operatorname{Im} \sqrt{\left(\zeta-f_{1}\right)\left(\zeta-f_{2}\right)}=d_{t} \xi_{2}+\frac{d_{t}\left(f_{2}-f_{1}\right)^{2}}{8} \rho^{-1} \sin \varphi+O\left(\rho^{-2}\right)
$$

при $\rho \rightarrow \infty$. В свою очередь, из (6.10)-(6.12) вытекает, что

$$
\psi_{2 t+2, t}(x)=\frac{d_{t}(s)\left(f_{2}(s)-f_{1}(s)\right)^{2}}{8} r^{-1} \sin \varphi+o\left(r^{-1}\right), \quad d_{0}(s)=c_{01}(s) .
$$

Из (6.10), (6.13) следует, что

$$
d_{t}(s)=\frac{1}{8^{t}} \mathscr{D}^{t} c_{01}(s)
$$

где $\mathscr{D}^{t}$ - линейный дифференциальный оператор второго порядка, определенньй в (1.7). В силу леммы 2.1, (6.13) и последнего равенства приходим к (1.6). Теорема доказана.

Обоснуем построенные асимптотики. В [36], [37] было доказано следующее утверждение. 
ЛЕмма 6.4. Пусть $\lambda_{0}$ - простое собственное значение предельной задачи (1.1). Тогда

а) для нормированных в $L_{2}(\Omega)$ собственных функций имеет место сходимость $\phi_{\varepsilon} \rightarrow \psi_{0}$ в $W_{2}^{1}(\Omega)$;

б) при $\lambda$, близких $к \lambda_{0}$, для решений краевой задачи

$$
-\Delta u_{\varepsilon}=\lambda u_{\varepsilon}+f \quad \text { в } \quad \Omega, \quad \frac{\partial u_{\varepsilon}}{\partial \tau}=0 \quad \text { на } \gamma_{\varepsilon}, \quad u_{\varepsilon}=0 \quad \text { на } \Gamma_{\varepsilon}
$$

имеет место представление

$$
u_{\varepsilon}=\frac{\phi_{\varepsilon}}{\lambda_{\varepsilon}-\lambda} \int_{\Omega} \phi_{\varepsilon} f d x+\widetilde{u}_{\varepsilon},
$$

где $\widetilde{u}_{\varepsilon}-$ голоморфная по $\lambda$ функция, ортогональная $\phi_{\varepsilon}$ в $L_{2}(\Omega)$, причем справедлива равномерная по є и $\lambda$ оченка

$$
\left\|\widetilde{u}_{\varepsilon}\right\|_{W_{2}^{1}(\Omega)} \leqslant C_{1}\|f\|_{L_{2}(\Omega)} .
$$

Пусть $\widehat{\lambda}_{\varepsilon, N}, \widehat{v}_{N}$ - соответственно частичные суммы рядов (1.4), (1.9), построенных в теореме 6.1.

Лемма 6.5. Пусть $F^{N}=\chi\left(r t \varepsilon^{-1 / 2}\right)\left(\Delta+\widehat{\lambda}_{\varepsilon, N}\right) \widehat{v}_{N}, t-$ произвольная фиксированная положительная постоянная. Тогда существует решение $\Phi^{N} \in$ $W_{2}^{1}(\Omega) \cap C^{\infty}\left(\bar{\Omega} \backslash \partial \gamma_{\varepsilon}\right)$ краевой задачи

$$
(\Delta-1) \Phi^{N}=F^{N}, \quad x \in \Omega, \quad \Phi^{N}=0, \quad x \in \Gamma_{\varepsilon}, \quad \frac{\partial \Phi^{N}}{\partial \tau}=0, \quad x \in \gamma_{\varepsilon},
$$

и имеет место оценка

$$
\left\|\Phi^{N}\right\|_{L_{2}(\Omega)}<C \varepsilon^{N_{1}}
$$

где $N_{1}=(N-1) / 2$ растет неограниченно вместе с $N$.

ДокаЗАТЕЛЬство. Пусть $f_{i} \in L_{2}(\Omega)$ с носителями, сосредоточенными в окрестности кривой $\gamma$. Предварительно рассмотрим следующую краевую задачу:

$$
\begin{gathered}
(\Delta-1) \Phi^{N}=-f_{0}+\frac{1}{J} \sum_{i=1}^{2} \frac{\partial f_{i}}{\partial y_{i}}+\frac{1}{J} \frac{\partial f_{3}}{\partial s}, \quad x \in \Omega, \\
\Phi^{N}=0, \quad x \in \Gamma_{\varepsilon}, \quad \frac{\partial \Phi^{N}}{\partial \tau}=-f_{2}, \quad x \in \gamma_{\varepsilon},
\end{gathered}
$$

где $J$ - якобиан перехода от переменных $x$ к переменньм $(y, s), W_{2,0}^{1}\left(\Omega ; \Gamma_{\varepsilon}\right)$ - замыкание в норме $W_{2}^{1}(\Omega)$ функций из $C^{\infty}(\bar{\Omega})$, обрашающихся в нуль в окрестности $\Gamma_{\varepsilon}$. Под решением краевой задачи (6.18) понимается элемент $\Phi^{N} \in W_{2,0}^{1}\left(\Omega ; \Gamma_{\varepsilon}\right)$, удовлетворяющий интегральному равенству

$$
\left(\nabla \Phi^{N}, \nabla w\right)_{\Omega}+\left(\Phi^{N}, w\right)_{\Omega}=\left(f_{0}, w\right)_{\Omega}+\sum_{i=1}^{2}\left(\frac{f_{i}}{J}, \frac{\partial w}{\partial y_{i}}\right)_{\Omega}+\left(\frac{f_{3}}{J}, \frac{\partial w}{\partial s}\right)_{\Omega}
$$


при любом $w \in W_{2,0}^{1}\left(\Omega ; \Gamma_{\varepsilon}\right)$. Правая часть этого равенства есть линейньй ограниченный функционал, заданный на $W_{2,0}^{1}\left(\Omega ; \Gamma_{\varepsilon}\right)$. Поэтому из теоремы Рисса следует существование и единственность решения краевой задачи (6.18). Если теперь $g_{i} \in C^{\infty}\left(\bar{\Omega} \backslash \partial \gamma_{\varepsilon}\right)$ и $g_{2}=0$ на $\gamma_{\varepsilon}$, то из теорем о повышении гладкости решений эллиптических краевых задач следует, что $\Phi^{N} \in W_{2}^{1}(\Omega) \cap C^{\infty}\left(\bar{\Omega} \backslash \partial \gamma_{\varepsilon}\right)$ удовлетворяет однородному граничному условию Неймана на $\gamma_{\varepsilon}$ в обычном (классическом) смысле. Полагая $w=\Phi^{N}$ в (6.19), получаем оценку

$$
\left\|\Phi^{N}\right\|_{W_{2}^{1}(\Omega)} \leqslant C \sum_{i=0}^{3}\left\|f_{i}\right\|_{L_{2}(\Omega)},
$$

где постоянная $C$ не зависит от $\varepsilon$.

Рассмотрим теперь краевую задачу (6.16). Так как $v_{k, q} \in \mathscr{F}_{k-2 q}^{(1)}$ являются решениями краевых задач (6.2), то, учитывая замечание 5.1 , определение множества $\mathscr{F}_{j}^{(1)}$ и определение оператора $\Delta($ см. $(4.1))$, имеем

$$
F^{N}=-f_{0}+\frac{1}{J} \sum_{i=1}^{2} \frac{\partial f_{i}}{\partial y_{i}}+\frac{1}{J} \frac{\partial f_{3}}{\partial s}
$$

где

$$
\begin{aligned}
& f_{0}=\chi\left(r t \varepsilon^{-1 / 2}\right) \sum_{i=1}^{N} \sum_{q=0}^{[(i-1) / 2]}\left(\ln ^{q} \varepsilon r^{i+2 q} \mathscr{P}^{(1)}(D) v_{N-i+1, q}+S(\varepsilon, \ln \varepsilon) v_{i, q}\right), \\
& f_{1}=\chi\left(r t \varepsilon^{-1 / 2}\right) \sum_{i=1}^{N} \sum_{q=0}^{[(i-1) / 2]} \ln ^{q} \varepsilon r^{i+2 q+1} \mathscr{P}^{(2)}(D) v_{N-i+1, q}, \\
& f_{2}=\chi\left(r t \varepsilon^{-1 / 2}\right) \sum_{i=1}^{N} \sum_{q=0}^{[(i-1) / 2]} \ln ^{q} \varepsilon r^{i+2 q+1} Q(\varepsilon, y, s) \frac{\partial v_{N-i+1, q}}{\partial y_{2}}, \\
& f_{3}=\chi\left(r t \varepsilon^{-1 / 2}\right) \sum_{i=1}^{N} \sum_{q=0}^{[(i-1) / 2]} \ln ^{q} \varepsilon r^{i+2 q} \mathscr{P}^{(3)}(D) v_{N-i+1, q}, \\
& \mathscr{P}^{(k)}(D)=\mathscr{P}^{(k)}(D, y, s, \varepsilon)-\text { полиномы первого порядка относительно символа } \\
& \text { дифференцирования } D=\left(\frac{\partial}{\partial y_{1}}, \frac{\partial}{\partial y_{2}}, \frac{\partial}{\partial s}\right) \text { с бесконечно дифференцируемыми по }
\end{aligned}
$$
$y$ и $s$ коэффициентами, $Q(\varepsilon, y, s)$ - бесконечно дифференцируемая по $y$ и $s$ функция, $S(\varepsilon, \ln \varepsilon)$ - многочлен по $\varepsilon$ и $\ln \varepsilon$, причем максимальная степень по $\ln \varepsilon$ равна $[(N-1) / 2]$. Так как $v_{k, q}$ удовлетворяют однородному граничному условию Неймана на $\gamma_{\varepsilon}$, то $f_{2}=0$ на $\gamma_{\varepsilon}$. Так как $f_{i} \in L_{2}(\Omega)$ с носителями, сосредоточенными в окрестности кривой $\gamma$, то краевая задача (6.16) разрешима. Из (6.21) с учетом того, что носитель $\chi\left(r t \varepsilon^{-1 / 2}\right)$ лежит в области $r<2 t^{-1} \varepsilon^{1 / 2}$, получаем

$$
\left\|f_{i}\right\|_{L_{2}(\Omega)}=O\left(\varepsilon^{(N-1) / 2}\right), \quad \rho \rightarrow \infty .
$$

Из (6.20), (6.22) следует справедливость (6.17).

Обозначим через $\widehat{\Psi}_{\varepsilon, N}$ частичную сумму ряда (1.8), построенного в теореме 6.1,

$$
\widehat{\psi}_{\varepsilon}(x)=\left(1-\chi\left(r t \varepsilon^{-1 / 2}\right)\right) \widehat{\Psi}_{\varepsilon, N}(x)+\chi\left(r t \varepsilon^{-1 / 2}\right) \widehat{v}_{N}\left(y \varepsilon^{-1}, s, \varepsilon\right)+\Phi^{N}(x, \varepsilon),
$$

где $\Phi^{N}$ - решение краевой задачи (6.16). 
Теорема 6.2. Функиия $\widehat{\psi}_{\varepsilon} \in W_{2}^{1}(\Omega) \cap C^{\infty}\left(\bar{\Omega} \backslash \partial \gamma_{\varepsilon}\right)$ является решением краевой задачи

$$
-\left(\Delta+\lambda_{\varepsilon, N}\right) \widehat{\psi}_{\varepsilon}=\widehat{f}_{\varepsilon}, \quad x \in \Omega, \quad \widehat{\psi}_{\varepsilon}=0, \quad x \in \Gamma_{\varepsilon}, \quad \frac{\partial \widehat{\psi}_{\varepsilon}}{\partial \tau}=0, \quad x \in \gamma_{\varepsilon},
$$

¿де $\left\|\widehat{f}_{\varepsilon}\right\|_{L_{2}(\Omega)}<c_{N_{2}} \varepsilon^{N_{2}},\left\|\widehat{\psi}_{\varepsilon}\right\|_{L_{2}(\Omega)}=1+o(1)$ nрu $\varepsilon \rightarrow 0, \quad N_{2}$ растет неограниченно вместе $c N$.

ДокАЗАТЕльство. По определению функция $\widehat{\psi}_{\varepsilon} \in W_{2}^{1}(\Omega) \cap C^{\infty}\left(\bar{\Omega} \backslash \partial \gamma_{\varepsilon}\right)$ и удовлетворяет граничным условиям в (6.24). Верно равенство $\left\|\widehat{\psi}_{\varepsilon}\right\|_{L_{2}(\Omega)}=1+o(1)$ при $\varepsilon \rightarrow 0$. Подставляя $\widehat{\psi}_{\varepsilon}$ в уравнение $(6.24)$, получаем

$$
\begin{gathered}
\widehat{f}_{\varepsilon}=-\sum_{j=1}^{3} f_{\varepsilon, j}(x), \\
f_{\varepsilon, 1}(x)=\Delta \chi\left(r t \varepsilon^{-1 / 2}\right)\left(\widehat{v}_{N}-\widehat{\Psi}_{\varepsilon, N}\right)+2 \sum_{j=1}^{3} \chi\left(r t \varepsilon^{-1 / 2}\right)_{x_{j}}\left(\widehat{v}_{N}-\widehat{\Psi}_{\varepsilon, N}\right)_{x_{j}}, \\
f_{\varepsilon, 2}(x)=\left(1-\chi\left(r t \varepsilon^{-1 / 2}\right)\right)\left(\Delta+\widehat{\lambda}_{\varepsilon, N}\right) \widehat{\Psi}_{\varepsilon, N}, \\
f_{\varepsilon, 3}(x)=\left(1+\widehat{\lambda}_{\varepsilon, N}\right) \phi .
\end{gathered}
$$

Так как $\psi_{2+i, j} \in \mathscr{A}_{i+1-2 j}$, то в силу (6.6) при $t^{-1} \varepsilon^{1 / 2}<r<2 t^{-1} \varepsilon^{1 / 2}$ имеем

$$
\begin{aligned}
& \widehat{v}_{N}=K_{N}\left(\widehat{\Psi}_{\varepsilon, N}\right)+O\left(\varepsilon \rho^{-N}+\varepsilon^{N-1} \rho\right), \quad \rho \rightarrow \infty, \\
& \widehat{\Psi}_{\varepsilon, N}=K_{N}\left(\widehat{\Psi}_{\varepsilon, N}\right)+O\left(r^{N+1}+\varepsilon^{N-1} r\right), \quad r \rightarrow 0 .
\end{aligned}
$$

Отсюда следует, что

$$
\widehat{\Psi}_{\varepsilon, N}-\widehat{v}_{N}=O\left(r^{N+1}+\varepsilon^{N-1} r+\varepsilon \rho^{-N}+\varepsilon^{N-1} \rho\right)
$$

при $r \rightarrow 0, \rho \rightarrow \infty$. Так как supp $f_{\varepsilon, 1} \subset S_{2 t^{-1} \varepsilon^{1 / 2}}^{2} \backslash S_{t^{-1} \varepsilon^{1 / 2}}^{2}$, то из (6.25) вытекает, что $\left\|f_{\varepsilon, 1}\right\|_{L_{2}(\Omega)}=O\left(\varepsilon^{N_{3}}\right)$, где $N_{3}$ растет неограниченно вместе с $N$.

В силу того что $\psi_{i+2, j} \in \mathscr{A}_{i+1-2 j}$ являются решениями краевых задач (6.1) и $\psi_{i+2, j}=O\left(r^{i+1-2 j}\right)$ при $r \rightarrow 0$, имеем

$$
f_{\varepsilon, 2}(x)=\left(1-\chi\left(r t \varepsilon^{-1 / 2}\right)\right) \varepsilon^{N+1} \sum_{i=2}^{N} \sum_{q=0}^{[i / 2]} S_{i, q}^{(1)}(\varepsilon, \ln \varepsilon) \psi_{i, q}
$$

где $S_{i, q}^{(k)}$ - многочлены от $\varepsilon$ и $\ln \varepsilon$. Так как $\operatorname{supp} f_{\varepsilon, 2} \subset \Omega \backslash S_{t^{-1} \varepsilon^{1 / 2}}^{2}$, то $\left\|f_{\varepsilon, 2}\right\|_{L_{2}(\Omega)}=$ $O\left(\varepsilon^{N_{4}}\right)$, где $N_{4}$ растет неограниченно вместе с $N$.

Так как supp $f_{\varepsilon, 3} \subset \Omega \cap S_{2 t^{-1} \varepsilon^{1 / 2}}^{2}$, то из леммы 6.5 следует, что $\left\|f_{\varepsilon, 3}\right\|_{L_{2}(\Omega)}=$ $O\left(\varepsilon^{N_{1}}\right)$, где $N_{1}$ растет неограниченно вместе с $N$.

Из оценок для $f_{\varepsilon, j}$ получаем, что $\left\|\widehat{f}_{\varepsilon}\right\|_{L_{2}(\Omega)}<c_{N_{2}} \varepsilon^{N_{2}}$, где $N_{2}$ растет неограниченно вместе с $N$. Теорема доказана. 
Из (6.14), (6.15) легко выводится оценка

$$
\left\|u_{\varepsilon}\right\|_{W_{2}^{1}(\Omega)} \leqslant \frac{C}{\left|\lambda_{\varepsilon}-\lambda\right|}\|f\|_{L_{2}(\Omega)} .
$$

Применяя (6.26) к $u_{\varepsilon}=\widehat{\psi}_{\varepsilon}, \lambda=\widehat{\lambda}_{\varepsilon, N}, f=\widehat{f}_{\varepsilon}$, в силу теоремы 6.2 имеем, что $\left|\lambda_{\varepsilon}-\widehat{\lambda}_{\varepsilon, N}\right|=O\left(\varepsilon^{N_{2}}\right)$. Отсюда в силу произвола в выборе $N$ следует, что асимптотический ряд (1.4), удовлетворяющий утверждению теоремы 6.1, совпадает с асимптотикой собственного значения $\lambda_{\varepsilon}$, сходяшегося к $\lambda_{0}$ при $\varepsilon \rightarrow 0$.

Из леммы 6.4 следует, что $\widehat{\psi}_{\varepsilon}$ может быть представлена в виде

$$
\widehat{\psi}_{\varepsilon}=\widehat{c}_{\varepsilon} \phi_{\varepsilon}+\widetilde{u}_{\varepsilon}, \quad\left\|\widetilde{u}_{\varepsilon}\right\|_{W_{2}^{1}(\Omega)} \leqslant C_{1}\left\|\widehat{f}_{\varepsilon}\right\|_{L_{2}(\Omega)},
$$

где $\phi_{\varepsilon}$-нормированная в $L_{2}(\Omega)$ собственная функция краевой задачи (1.2). Обозначим $\widetilde{\psi}_{\varepsilon}=\widehat{c}_{\varepsilon} \phi_{\varepsilon}$. Так как $\widetilde{\psi}_{\varepsilon}-$ собственная функция, соответствующая $\lambda_{\varepsilon}$, а в силу (6.27) и теоремы $6.2\left\|\widehat{\psi}_{\varepsilon}-\widetilde{\psi}_{\varepsilon}\right\|_{W_{2}^{1}(\Omega)}=O\left(\varepsilon^{N_{2}}\right)$, где $N_{2}$ растет неограниченно

вместе с $N$, то из произвола в выборе $N$ следует справедливость (1.8) в $\Omega \backslash S_{t^{-1} \varepsilon^{1 / 2}}^{2}$ и справедливость (1.9) в $\Omega \cap S_{2 t^{-1} \varepsilon^{1 / 2}}^{2}$. Полагая $t=2$, получаем, что асимптотика (1.8) справедлива в $\Omega \backslash S_{\varepsilon^{1 / 2}}^{2}$, а полагая $t=1 / 2$, получаем, что асимптотика (1.9) справедлива в $\Omega \cap S_{2 \varepsilon^{1 / 2}}^{2}$. Теорема 1.1 доказана.

\section{§7. Доказательство теоремы 1.2}

Будем строить асимптотику $\lambda^{\varepsilon}$ в виде (1.4), а асимптотики $\psi^{\varepsilon}$ - в виде (1.8) при $\Omega_{\varepsilon} \backslash S_{\varepsilon^{1 / 2}}^{2}$ и (1.11) при $\Omega_{\varepsilon} \cap S_{2 \varepsilon^{1 / 2}}^{2}$. Подставляя (1.4), (1.8), (1.11), (4.1) в (1.3), получаем краевые задачи для коэффициентов рядов (1.8), (1.11):

$$
\begin{gathered}
-\left(\Delta+\lambda_{0}\right) \psi_{2+k, q}=\lambda_{2+k, q} \psi_{0}+\sum_{i=0}^{k-2} \sum_{j=0}^{q} \lambda_{k-i, q-j} \psi_{2+i, j} \quad \text { в } \Omega \backslash \gamma \\
\psi_{2+k, q}=0 \quad \text { на } \Gamma \\
\Delta_{\xi} v_{1+k, q}=-\lambda_{0} v_{k-1, q}-\sum_{i=0}^{k-3} \sum_{j=0}^{q} \lambda_{k-i-1, q-j} v_{i, j}+\sum_{i=1}^{k-2 q+1} R_{i, 2}(\xi, s, D) v_{k-i+1, q} \\
+\sum_{i=0}^{k-2 q} R_{i, 1}(\xi, s, D) v_{k-i, q}+\sum_{i=0}^{k-2 q-1} R_{i, 0}(\xi, s, D) v_{k-i-1, q} \quad \text { в } \mathbb{R}^{2} \backslash \bar{\omega}, \\
\frac{\partial v_{1+k, q}}{\partial \nu}=\sum_{i=1}^{k-1} p_{i}\left(l_{\xi}, s\right) \frac{\partial v_{k-i, q}}{\partial s} \quad \text { на } \partial \omega .
\end{gathered}
$$

Здесь и далее будем считать равными нулю $\lambda_{k, q}, \psi_{k, q}, v_{k, q}$, индексы у которых не являются членами рядов (1.4), (1.8), (1.11).

Из определения $K_{N}, \mathscr{B}_{i}^{(0)}$ и $\widetilde{\mathscr{G}}_{i}$ вытекает следуюший аналог леммы 6.1. 
Лемма 7.1. Пусть $\psi_{2+k, q} \in \mathscr{B}_{k+1-2 q}^{(0)} \cdot$ Тогда

$$
K_{\infty}\left(\Psi_{\varepsilon}(x)\right)=\sum_{i=0}^{\infty} \sum_{j=0}^{[i / 2]} \varepsilon^{i} \ln ^{j} \varepsilon V_{i, j}(\xi ; s), \quad \text { əдe } V_{i, j} \in \widetilde{\mathscr{G}}_{i-2 j}
$$

Еслик тому жее $\psi_{2+k, q}-$ решения (7.1), то ряды $V_{i, j}$ являются формальньми асимптотическими решениями уравнений (7.2) при $\rho \rightarrow \infty$, дде в правой части функиии $v_{i, j}$ заменены на рядь $V_{i, j}$.

Будем искать $\psi_{2+k, q}, \lambda_{2+k, q}$ в виде

$$
\psi_{2+k, q}(x)=\sum_{j=2 q}^{k+1} \Psi_{2+k, q}^{(j)}, \quad \lambda_{2+k, q}(x)=\sum_{j=2 q}^{k+1} \Lambda_{2+k, q}^{(j)} .
$$

Далее будем считать равными нулю $\Psi_{k, q}^{(m)}, \Lambda_{k, q}^{(n)}$, индексы у которых не являются членами (7.4). С учетом определения множества $\mathscr{B}_{i}^{(0)}$ аналогично лемме 6.2 доказывается

ЛЕмМа 7.2. Если $\Psi_{2+k, q}^{(t)} \in \mathscr{B}_{k+1-t}^{(0)}$, то функиия $\psi_{2+k, q}$, определенная равенством (7.4), принадлежст $\mathscr{B}_{k+1-2 q}^{(0)}$. Если к тому жсе $\Psi_{2+k, q}^{(t)}$ являются решениями краевьх задач

$$
\begin{gathered}
-\left(\Delta+\lambda_{0}\right) \Psi_{2+k, q}^{(t)}=\Lambda_{2+k, q}^{(t)} \psi_{0}+\sum_{i=0}^{k-2} \sum_{j=0}^{q} \sum_{m+n=t} \Lambda_{k-i, q-j}^{(m)} \Psi_{2+i, j}^{(n)} \quad n p u \quad x \in \Omega \backslash \gamma \\
\Psi_{2+k, q}^{(t)}=0 \quad \text { npu } x \in \Gamma,
\end{gathered}
$$

то $\psi_{2+k, q}$ являются решениями краевых задач (7.1).

Обозначим

$$
\begin{aligned}
& \psi^{(N)}(x, \varepsilon)=\psi_{0}(x)+\sum_{i=0}^{\infty} \sum_{j=0}^{\left[\frac{1}{2} \min \{N, i\}\right]} \varepsilon^{2+i} \ln ^{j} \varepsilon \psi_{2+i, j}^{(N)}(x), \\
& \psi^{(-1)}(x, \varepsilon)=\psi_{0}(x), \quad \psi_{2+k, j}^{(N)}(x)=\sum_{t=2 j}^{\min \{N, k+1\}} \Psi_{2+k, j}^{(t)}(x) .
\end{aligned}
$$

ЗАмечАние 7.1. Так как $\Psi_{2+k, q}^{(j)} \in \mathscr{B}_{k+1-j}$, то из определений $K_{N}, \psi^{(N)}(x, \varepsilon)$ следует, что $K_{N}\left(\psi^{(N-1)}\right)=K_{N}\left(\Psi_{\varepsilon}\right)$.

Из лемм 7.1, 7.2 вытекает следующий аналог леммы 6.3. 
ЛЕмма 7.3. Пусть $\Psi_{2+k, q}^{(j)} \in \mathscr{B}_{k+1-j}^{(0)}$. Тогда рядь $V_{i, j}$ в (7.3) имеют вид

$$
\begin{gathered}
V_{i, j}(\xi ; s)=\widetilde{V}_{i, j}(\xi ; s)+\left(1-\delta_{i}^{2 j}\right)\left(1-\delta_{i}^{2 j+1}\right)\left(g^{(i, j)}(s) \ln \rho+\widetilde{g}^{(i, j)}(s)\right) \\
+\left(1-\delta_{i}^{2 j}\right) \sum_{t=1}^{\infty} Z_{t}^{(i, j)}(\xi ; s) \rho^{-2 t}
\end{gathered}
$$

$Z_{t}^{(i, j)}(y ; s) r^{-2 t}-$ главный член асимптотики $\Psi_{t+i, j}^{(i-1)}$ в нуле, $g^{(i, j)}(s) \ln r+$ $\widetilde{g}^{(i, j)}(s)$ - главные члены асимптотики $\Psi_{i, j}^{(i-1)}$ в нуле,

$$
\widetilde{V}_{i, j}(\xi ; s)=\varepsilon^{-i} \ln ^{-j} \mathscr{K}_{i, j}\left(\psi^{(i-2)}(x, \varepsilon)\right), \quad \widetilde{V}_{i, j} \in \widetilde{\mathscr{G}}_{i-2 j},
$$

и, в частности, $\widetilde{V}_{0,0}(\xi ; s)=P_{0}(s), \quad \widetilde{V}_{1,0}(\xi ; s)=P_{1}(\xi ; s), \quad \widetilde{V}_{2,1}(\xi ; s)=g^{(2,0)}(s)$.

Если к тому же $\Psi_{2+k, q}^{(t)}$ являются решениями (7.5), то ряды $\widetilde{V}_{1+k, q}$ являются формальными асимптотическими решениями (7.2) при $\rho \rightarrow \infty$, где в правой части функции $v_{1+i, j}$ заменень на $V_{1+i, j}$.

Обозначим через $\widehat{v}_{N}(\xi, s, \varepsilon)$ частичную сумму ряда (1.11).

Tеорема 7.1. Существуют ряды (1.4), (1.8) и (1.11) такие, что

1) выполняется равенство (1.10);

2) функции $\psi_{2+i, q} \in \mathscr{B}_{i+1-2 q}^{(0)}$ являются решениями (7.1);

3) функиии $v_{i, q} \in \mathscr{G}_{i-2 q}$ являются решениями (7.2);

4) для любого $N \geqslant 0$ выполняется равенство (6.6).

ДокАЗАТЕЛЬСТво. В $\S 3$ было доказано, что сушествуют решения $v_{0,0}=v_{0}$, $v_{1,0}=v_{1}$ краевых задач $(7.2)$, определенные равенствами $(3.11),(3.19)$ с асимптотиками при $\rho \rightarrow \infty$

$$
v_{0,0}(\xi ; s)=\widetilde{V}_{0,0}(\xi ; s), \quad v_{1,0}(\xi ; s)=\widetilde{V}_{1,0}(\xi ; s)+\sum_{i=1}^{\infty} Z_{i}(\xi ; s) \rho^{-2 i},
$$

причем $Z_{1}(\xi ; s)=\rho\left(A_{1}(s) \sin \varphi+B_{1}(s) \cos \varphi\right)$, где $A_{1}, B_{1}$ определяются в (3.21). Из определений $\mathscr{G}_{q}$ и $v_{i, j}$ следует, что $v_{0,0} \in \mathscr{G}_{0}, v_{1,0} \in \mathscr{G}_{1}$. Выберем главные члены $Z_{i}^{(1,0)}(y ; s) r^{-2 i}$ асимптотик $\Psi_{i+1,0}^{(0)}(x)$ равными $Z_{i}(y ; s) r^{-2 i}$ из (7.6). Из (7.6) и леммы 7.3 следует, что

$$
v_{0,0}(\xi ; s)=V_{0,0}(\xi ; s), \quad v_{1,0}(\xi ; s)=V_{1,0}(\xi ; s), \quad \rho \rightarrow \infty .
$$

С другой стороны, в силу леммы 4.9 существуют постоянные $\Lambda_{i+1,0}^{(0)}$ и функции $\Psi_{i+1,0}^{(0)} \in \mathscr{B}_{i}^{(0)}$ с заданными главными членами асимптотик такие, что (7.5) выполняется при $t=0$. В силу леммы 3.2 имеем

$$
\begin{aligned}
& \Psi_{2,0}^{(0)}=U^{(1)}\left(x ; A_{1}(s), B_{1}(s)\right), \\
& \Lambda_{2,0}^{(0)}=-2 \pi \int_{\gamma}\left(c_{10} B_{1}+c_{01} A_{1}\right) d s .
\end{aligned}
$$


Таким образом, построен ряд $\psi^{(0)}(x, \varepsilon)$. Так как $K_{1}\left(\psi^{(0)}\right)=K_{1}\left(\Psi_{\varepsilon}\right)$ (замечание 7.1), то (6.6) выполняется для $N=1$.

Так как $\widetilde{V}_{2,0} \in \widetilde{\mathscr{G}}_{2}$, то в силу леммы 5.3 сушествует решение $v_{2,0} \in \mathscr{G}_{2}$ задачи (7.2) с асимптотикой

$$
v_{2,0}(\xi ; s)=\widetilde{V}_{2,0}(\xi ; s)+g^{(2,0)}(s) \ln \rho+\widetilde{g}^{(2,0)}(s)+\sum_{i=1}^{\infty} Z_{i}(\xi ; s) \rho^{-2 i}, \quad \rho \rightarrow \infty,
$$

где $\widetilde{g}^{(2,0)}(s) \in C^{\infty}(\gamma)$ - произвольная функция. По построению $\widetilde{v}=v_{2}-v_{2,0}$ является гармонической функцией, удовлетворяющей однородным граничньм условиям Неймана на $\partial \omega$, с асимптотикой $\widetilde{v}=X_{2}+X_{1}+(G(s)-g(s)) \ln \rho+O(1)$ при $\rho \rightarrow \infty$, где $v_{2}$ построена в $\S 3, X_{k}(\xi ; s)$ - гармонический полином степени $k$. Из леммы 3.1 следует, что $\widetilde{v}=X_{2}+X_{1}+O(1)$ при $\rho \rightarrow \infty$. Следовательно, $g^{(2,0)}(s)=G(s)$.

Выберем главный член асимптотики $\Psi_{2,0}^{(1)}(x)$ равным $G(s) \ln r$ и главные члены $Z_{i}^{(2,0)}(y ; s) r^{-2 i}$ асимптотик $\Psi_{i+2,0}^{(1)}(x)$ равными $Z_{i}(y ; s) r^{-2 i}$ из (7.8). Далее, в силу лемм $4.9,4.10$ сушествуют постоянные $\Lambda_{i+2,0}^{(1)}$ при $i \geqslant 0$ и функции $\Psi_{i+2,0}^{(1)} \in \mathscr{B}_{i}^{(0)}$ с заданными главными членами асимптотик и такие, что (7.5) выполняется при $t=1$. В силу леммы 3.2 имеем $\Psi_{2,0}^{(1)}=U^{(0)}(x ; G(s)), \Psi_{2,0}^{(1)}=G(s) \ln r+\widetilde{G}(s)+o(1)$ при $r \rightarrow 0$ и

$$
\Lambda_{2,0}^{(1)}=2 \pi \int_{\gamma} c_{00} G d s
$$

Выбирая теперь в $(7.8) \widetilde{g}^{(2,0)}(s)=\widetilde{G}(s)$, в силу леммы 7.3 получаем

$$
v_{2,0}(\xi ; s)=V_{2,0}(\xi ; s), \quad \rho \rightarrow \infty .
$$

Из (7.7), (7.9) следует (1.10).

Легко видеть, что $v_{2,1}(\xi ; s)=\widetilde{V}_{2,1}(\xi ; s)=G(s)$ является решением краевой задачи (7.2). Таким образом, построен ряд $\psi^{(1)}(x, \varepsilon)$. Так как $K_{2}\left(\psi^{(1)}\right)=K_{2}\left(\Psi_{\varepsilon}\right)$ (замечание 7.1), то (6.6) вьполняется для $N=2$.

Дальнейшее доказательство проводится по индукции аналогично теореме 6.1.

В работах [36], [38] доказаны следуюшие утверждения.

ЛЕмма 7.4. Пусть $\lambda_{0}$ - простое собственное значение предельной задачи (1.1). Тогда

а) для нормированных в $L_{2}\left(\Omega_{\varepsilon}\right)$ собственных функиий имеет место сходимость $\left\|\phi^{\varepsilon}-\psi_{0}\right\|_{W_{2}^{1}\left(\Omega_{\varepsilon}\right)} \rightarrow 0$

б) при $\lambda$, близких к $\lambda_{0}$, для решений краевой задачи

$$
-\Delta u^{\varepsilon}=\lambda u^{\varepsilon}+f \quad \text { в } \Omega_{\varepsilon}, \quad \frac{\partial u^{\varepsilon}}{\partial \tau}=0 \quad \text { на } \partial \sigma_{\varepsilon}, \quad u^{\varepsilon}=0 \quad \text { на } \Gamma
$$

справедлива равномерная по є и $\lambda$ оценка

$$
\left\|u^{\varepsilon}\right\|_{W_{2}^{1}\left(\Omega_{\varepsilon}\right)} \leqslant \frac{C}{\left|\lambda^{\varepsilon}-\lambda\right|}\|f\|_{L_{2}\left(\Omega_{\varepsilon}\right)} .
$$


Пусть $\widehat{\lambda}_{\varepsilon, N}, \widehat{\Psi}_{\varepsilon, N}, \widehat{v}_{N}-$ соответственно частичные суммы рядов (1.4), (1.8), (1.11), построенных в теореме 7.1. Обозначим

$$
\widehat{\psi}^{\varepsilon}(x)=\left(1-\chi\left(r t \varepsilon^{-1 / 2}\right)\right) \widehat{\Psi}_{\varepsilon, N}(x)+\chi\left(r t \varepsilon^{-1 / 2}\right) \widehat{v}_{N}\left(y \varepsilon^{-1}, s, \varepsilon\right),
$$

$t$ - произвольная фиксированная положительная постоянная. Аналогично теореме 6.2 доказывается

ТЕОРема 7.2. Функиия $\widehat{\psi}^{\varepsilon} \in C^{\infty}\left(\bar{\Omega}_{\varepsilon}\right)$ является решением краевой задачи

$$
-\left(\Delta+\lambda_{\varepsilon, N}\right) \widehat{\psi}^{\varepsilon}=\widehat{f}_{\varepsilon}, \quad x \in \Omega_{\varepsilon}, \quad \frac{\partial \widehat{\psi}^{\varepsilon}}{\partial \tau}=0, \quad x \in \partial \sigma_{\varepsilon}, \quad \widehat{\psi}^{\varepsilon}=0, \quad x \in \Gamma
$$

əде $\left\|\widehat{f}_{\varepsilon}\right\|_{L_{2}(\Omega)}<c_{N} \varepsilon^{N_{1}},\left\|\widehat{\psi}^{\varepsilon}\right\|_{L_{2}(\Omega)}=1+o(1)$ nри $\varepsilon \rightarrow 0, N_{1}$ растет неограниченно вместе $c N$.

Обоснование асимптотик полностью аналогично обоснованию асимптотик краевой задачи (1.3), только вместо теоремы 6.1 используется теорема 7.1, а вместо леммы 6.4 - лемма 7.4 .

В заключение автор хотела бы выразить благодарность Р.Р. Гадыльшину и Д. И. Борисову за постоянное внимание к работе и полезные замечания.

\section{Список литературы}

1. Ван-Дайк М. Методы возмущений в механике жидкости. М.: Мир, 1967.

2. Бабич B. М., Булдьрев В. С. Асимптотические методы в задачах дифрракции коротких волн. М.: Наука, 1972.

3. Марченко B. A., Хруслов E. Я. Краевые задачи в областях с мелкозернистой границей. Киев: Наукова думка, 1974.

4. Санчес-Паленсиа Е. Неоднородные среды и теория колебаний. М.: Мир, 1984.

5. Бахвалов Н. С., Панасенко Г. П. Осреднение процессов в периодических средах. Математические задачи механики композиционных материалов. М.: Наука, 1984.

6. Ильин A. М. Согласование асимптотических разложений решений краевых задач. М.: Наука, 1989.

7. Олейник О.А., Иосифьян Г. А., Шамаев А. С. Математические задачи теории сильно неоднородных упругих сред. М.: Изд-во МГУ, 1990.

8. Мазъя В.Г., Назаров С.А., Пламеневский Б. А. Асимптотика решений эллиптических краевых задач при сингулярном возмущении области. Тбилиси: Изд-во Тбилисского ун-та, 1981.

9. Ильин А. М. Краевая задача для эллиптического уравнения второго порядка в области с узкой щелью. І. Двумерньй случай // Матем. сб. 1976. Т. 99(141). С. 514-537.

10. Ильин А. М. Краевая задача для эллиптического уравнения второго порядка в области с узкой щелью. II. Область с мальм отверстием // Матем. сб. 1977. Т. $103(145)$. C. $265-284$.

11. Федорюк M. В. Задача Дирихле для оператора Лапласа во внешности тонкого тела вращения // Труды сем. им. С. Л. Соболева. №1. Новосибирск: Ин-т математики СО АН CCCP, 1980. C. 113-131.

12. Ильин A. М. Исследование асимптотики решения эллиптической краевой задачи в области с мальм отверстием // Труды сем. им. И.Г. Петровского. №6. М.: Изд-во МГУ, 1981. C. $57-82$.

13. Чечкин $Г$. А. Усреднение краевых задач с сингулярным возмущением граничных условий // Матем. сб. 1993. Т. 184. №6. С. 99-150. 
14. Аргатов И. И., Назаров С. А. Асимптотическое решение задачи Синьорини с препятствием на тонком продолговатом множестве // Матем. сб. 1996. Т. 187. № 10. С. 3-32.

15. Назаров C.A., Паукито М.В. Дискретные модели и осреднение в задачах теории упругости. Л.: ЛГУ, 1984.

16. Самарский $A$. A. О влиянии закрепления на собственные частоты замкнутых объемов // Докл. АН СССР. 1948. Т. 63. С. 631-634.

17. Swanson C. A. Asymptotic variational formulae for eigenvalues // Canad. Math. Bull. 1963. V. 6. № 1. P. 15-25.

18. Днестровский Ю. Н. Об изменении собственных чисел при изменении границы областей // Вестн. МГУ. Сер. 1. Матем., мех. 1964. №9. С. 61-74.

19. Ozawa $S$. Singular variation of domains and eigenvalues of the Laplacian // Duke Math. J. 1981. V. 48. P. 767-778.

20. Мазья В. Г., Назаров С. П., Пламеневский Б. А. Асимптотические разложения собственных чисел краевых задач для оператора Лапласа в областях с мальми отверстиями // Изв. АН СССР. Сер. матем. 1984. Т. 48. С. 347-371.

21. Гадыльшин P.P., Ильин А. М. Асимптотика собственных значений задачи Дирихле в области с узкой щелью // Матем. сб. 1998. Т. 189. №4. С. 25-48.

22. Jimbo $S$. The singularity perturbed domain and the characterization for the eigenfunctions with Neumann boundary condition // J. Differential Equations. 1989. V. 77. P. 322-350.

23. Hempel R., Seco L., Simon B. The essential spectrum of Neumann Laplacians on some bounded singular domains // J. Funct. Anal. 1991. V. 102. P. 448-483.

24. Гадыльиин Р. Р. Асимптотика второй собственной частоты для системы двух тел, соединенных тонкой перемычкой // ТМФ. 1993. Т. 97. № 1. С. 68-77.

25. Гадыльиин Р. Р. О собственных частотах тел с тонкими отростками. II. Асимптотики // Матем. заметки. 1994. Т. 55. №1. С. 20-34.

26. Arrieta J. M. Neumann eigenvalue problems on exterior perturbations of the domain // J. Differential Equations. 1995. V. 118. P. 54-103.

27. Гадыльиин Р. Р. Расщепление кратного собственного значения в краевой задаче для мембраны, закрепленной на малом участке границы // Сиб. матем. журн. 1993. Т. 34. № 3. C. $43-61$.

28. Гадыльиин Р. Р. Асимптотика собственного значения сингулярно возмущенной эллиптической задачи с мальм параметром в граничном условии // Дифференц. уравнения. 1986. T. 22. № 4. C. 640-652.

29. Гадыльиин Р. Р. Расщепление кратного собственного значения задачи Дирихле для оператора Лапласа при сингулярном возмущении граничного условия // Матем. заметки. 1992. Т. 52. № 4. С. 42-55.

30. Гадыльиин Р. Р. О возмущении спектра лапласиана при смене типа граничного условия на малой части границы // ЖВМиМФ. 1996. Т. 36. № 7. С. 77-88.

31. Гадыльиин Р. Р. Об асимптотике собственных значений для периодически закрепленной мембраны // Алгебра и анализ. 1998. Т. 10. №1. С. 3-19.

32. Гадыльиин P. P. Асимптотики собственных значений краевой задачи с быстроосциллирующими граничньми условиями // Дифференц. уравнения. 1999. Т. 35. № 4. С. 540-551.

33. Борисов Д. И. О краевой задаче в цилиндре с частой сменой типа граничных условий // Матем. сб. 2002. Т. 193. № 7. С. 37-68.

34. Borisov D. I. On a model boundary value problem for Laplacian with frequently alternating type of boundary condition // Asymptot. Anal. 2003. V. 35. № 1. P. 1-26.

35. Борисов Д. И. Асимптотики и оценки собственных элементов лапласиана с частой непериодической сменой граничных условий // Изв. РАН. Сер. матем. 2003. Т. 67. №6. C. $23-70$.

36. Планида М. Ю. О сходимости решений сингулярно возмущенных краевых задач для лапласиана // Матем. заметки. 2002. Т. 71. №6. С. 867-877.

37. Планида М. Ю. Об асимптотике собственных значений для цилиндра, теплоизолированного на узкой полосе // ЖВМиМФ. 2003. Т. 43. № 3. С. 422-432. 
38. Планида М. Ю. Об асимптотике собственных значений лапласиана в области с граничным условием Неймана на вырезанной тонкой трубке // ЖВМиМФ. 2004. Т. 44 . № 4 . C. $717-730$.

39. Планида М. Ю. Асимптотики собственных элементов оператора Лапласа со сменой типа граничного условия на узкой уплощенной полосе // Матем. заметки. 2004. Т. 75. C. $236-252$.

40. Planida M. Yu. Asymptotics for eigenvalues of Laplacian with Neumann boundary condition on a thin tube cut out // C.R. Acad. Sci. Ser. Mechan. 2003. V. 331. P. 531-536.

41. Михлин С. Г. Линейные уравнения в частных производных. М.: Наука, 1976.

42. Лаврентьев М. А., Шабат Б. В. Методы теории функций комплексного переменного. М.: Наука, 1987.

43. Соболев С. Л. Некоторые применения функционального анализа в математической физике. М.: Наука, 1988.

44. Ладыженская О.А., Уральцева H. Н. Линейные и квазилинейные уравнения эллиптического типа. М.: Наука, 1977.

45. Михайлов В. П. Дифференциалњные уравнения в частных производных. М.: Наука, 1983.

Башкирский государственньй

Поступила в редакцию

педагогический университет, Уфа

17.05.2004

E-mail: planida@bspu.ru 